\title{
REGULARITY BELOW THE CONTINUOUS THRESHOLD IN A TWO-PHASE PARABOLIC FREE BOUNDARY PROBLEM
}

\author{
KAJ NYSTRÖM
}

\begin{abstract}
In this paper we study free boundary regularity in a parabolic two-phase problem below the continuous threshold. We consider unbounded domains $\Omega \subset \mathbf{R}^{n+1}$ assuming that $\partial \Omega$ separates $\mathrm{R}^{n+1}$ into two connected components $\Omega^{1}=\Omega$ and $\Omega^{2}=\mathrm{R}^{n+1} \backslash \bar{\Omega}$. We furthermore assume that both $\Omega^{1}$ and $\Omega^{2}$ are parabolic NTA-domains, that $\partial \Omega$ is Ahlfors regular and for $i \in\{1,2\}$ we define $\omega^{i}\left(\hat{X}^{i}, \hat{t}^{i}, \cdot\right)$ to be the caloric measure at $\left(\hat{X}^{i}, \hat{t}^{i}\right) \in \Omega^{i}$ defined with respect to $\Omega^{i}$. In the paper we make the additional assumption that $\omega^{i}\left(\hat{X}^{i}, \hat{t}^{i}, \cdot\right)$, for $i \in\{1,2\}$, is absolutely continuous with respect to an appropriate surface measure $\sigma$ on $\partial \Omega$ and that the Poisson kernels $k^{i}\left(\hat{X}^{i}, \hat{t}^{i}, \cdot\right)=$ $d \omega^{i}\left(\hat{X}^{i}, \hat{t}^{i}, \cdot\right) / d \sigma$ are such that $\log k^{i}\left(\hat{X}^{i}, \hat{t}^{i}, \cdot\right) \in \operatorname{VMO}(d \sigma)$. Our main result (Theorem 1) states that, under these assumptions, $C_{r}(X, t) \cap \partial \Omega$ is Reifenberg flat with vanishing constant whenever $(X, t) \in \partial \Omega$ and $\min \left\{\hat{t}^{1}, \hat{t}^{2}\right\}>t+4 r^{2}$. This result has an important consequence (Theorem 3 ) stating that if the two-phase condition on the Poisson kernels is fulfilled, $\Omega^{1}$ and $\Omega^{2}$ are parabolic NTA-domains and $\partial \Omega$ is Ahlfors regular then if $\Omega$ is close to being a chord arc domain with vanishing constant we can in fact conclude that $\Omega$ is a chord arc domain with vanishing constant.
\end{abstract}

\section{Introduction}

In this paper we study a free boundary regularity problem for a parabolic two-phase problem below the continuous threshold. We consider unbounded domains $\Omega \subset \mathbf{R}^{n+1}$ assuming that $\partial \Omega$ separates $\mathbf{R}^{n+1}$ into two connected components $\Omega^{1}=\Omega$ and $\Omega^{2}=\mathrm{R}^{n+1} \backslash \bar{\Omega}$ (this is made more precise in Definition 3 below). We furthermore assume that both $\Omega^{1}$ and $\Omega^{2}$ are parabolic NTA-domains and our notion of parabolic NTA-domains is defined at the beginning of section 2 below. As is described below the bounded continuous Dirichlet problem for the heat equation always has a unique solution in this type of domains. Let $(X, t), X=\left(x_{0}, \ldots, x_{n-1}\right), t \in \mathrm{R}$ denote a point in $\mathrm{R}^{n+1}$ and for given $r>0$ set $C_{r}(X, t)=\left\{(Y, s):|Y-X|<r,|t-s|<r^{2}\right\}$. For fixed $(\hat{X}, \hat{t}) \in \Omega$ we let $\omega(\hat{X}, \hat{t}, \cdot)$ denote the parabolic measure (in this paper this measure is refered to as the caloric measure) for the heat equation obtained from the maximum principle and the Riesz representation theorem. Let $\Delta(X, t, r)=C_{r}(X, t) \cap \partial \Omega$ whenever $(X, t) \in \partial \Omega$ and $r>0$. Given

Received November 24, 2005. 
$(\hat{X}, \hat{t}) \in \Omega$ let $(X, t) \in \partial \Omega$ and suppose $|X-\hat{X}|^{2} \leq A(\hat{t}-t)$ for some $A \geq 2$. In [11] it is proven that $\omega(\hat{X}, \hat{t}, \cdot)$ is, in the setting of parabolic NTA-domains, a doubling measure in the sense that there exists $a_{1}=a_{1}(n, A)$ such that if $\hat{t}-t \geq 8 r^{2}$ then

$$
\omega(\hat{X}, \hat{t}, \Delta(X, t, 2 r)) \leq a_{1} \omega(\hat{X}, \hat{t}, \Delta(X, t, r)) .
$$

For $i \in\{1,2\}$ we let $\left(\hat{X}^{i}, \hat{t}^{i}\right) \in \Omega^{i}$ and define $\omega^{1}\left(\hat{X}^{1}, \hat{t}^{1}, \cdot\right)$ and $\omega^{2}\left(\hat{X}^{2}, \hat{t}^{2}, \cdot\right)$ to be the caloric measures defined w.r.t. $\Omega^{1}$ and $\Omega^{2}$ respectively. For a Borel set $F \subset \mathrm{R}^{n+1}$ we let $\bar{F}, \partial F$ denote the closure and the boundary of $F$ respectively, and define $\sigma(F)=\int_{F} d \sigma_{t} d t$ where $d \sigma_{t}$ is $n-1$ dimensional Hausdorff measure on the time slice $F \cap\left(\mathrm{R}^{n} \times\{t\}\right)$.

Definition 1. Let $\Omega$ be a connected open set in $\mathbf{R}^{n+1}$. We say that $\partial \Omega$ satisfies a $(M, R)$ Ahlfors condition, $M \geq 4$, if for all $(X, t) \in \partial \Omega$ and $0<r \leq R$,

$$
\sigma\left(\partial \Omega \cap C_{r}(X, t)\right) \leq M r^{n+1} .
$$

Using the fact that Hausdorff measure does not increase under a projection we deduce that for $0<r \leq R,(X, t) \in \partial \Omega$,

$$
(r / 2)^{n+1} \leq \sigma\left(\partial \Omega \cap C_{r}(X, t)\right) \leq M r^{n+1},
$$

whenever $\partial \Omega$ separates $\mathrm{R}^{n+1}$ and satisfies a $(M, R)$ Ahlfors condition.

In the following we define a notion of two-sided NTA-domains with Ahlfors regular boundary. For the technical definition of our notion of parabolic NTAdomains we refer the reader to the beginning of section 2 below.

DEFINITION 2. If $\Omega$ is a connected open set in $\mathbf{R}^{n+1}$ such that $\partial \Omega$ separates $\mathrm{R}^{n+1}$ into two connected components $\Omega^{1}=\Omega$ and $\Omega^{2}=\mathrm{R}^{n+1} \backslash \bar{\Omega}$ and $\Omega^{1}$ and $\Omega^{2}$ are parabolic NTA-domains then we call $\Omega$ a two-sided NTA-domain. If, in addition, $\partial \Omega$ satisfies a $(M, R)$ Ahlfors for some $R>0$ then $\Omega$ is called a two-sided NTA-domain with Ahlfors regular boundary.

In the following we will assume that $\Omega$ is a two-sided NTA-domain with Ahlfors regular boundary and that $\omega^{i}\left(\hat{X}^{i}, \hat{t}^{i}, \cdot\right)$, for $i \in\{1,2\}$, is absolutely continuous with respect to the surface measure $\sigma$. We define Poisson kernels as $k^{i}\left(\hat{X}^{i}, \hat{t}^{i}, \cdot\right)=d \omega^{i}\left(\hat{X}^{i}, \hat{t}^{i}, \cdot\right) / d \sigma$. The regularity assumption that we will impose on the Poisson kernels is that $\log k^{i}\left(\hat{X}^{i}, \hat{t}^{i}, \cdot\right) \in \operatorname{VMO}(d \sigma)$ for $i \in\{1,2\} . \operatorname{VMO}(d \sigma)$ is the space of functions of vanishing mean oscillation defined w.r.t. the measure $d \sigma$. To properly define this space we let $a=a(\Delta(X, t, \rho), f)$ denote the average of $f=\log k^{i}\left(\hat{X}^{i}, \hat{t}^{i}, \cdot\right)$ on $\Delta(X, t, \rho)$ 
with respect to $\sigma$. Then we say that $f \in \operatorname{VMO}(d \sigma)$ provided for each compact $K \subset \partial \Omega \cap\left\{(Y, s): s<\hat{t}^{i}\right\}$,

$$
\lim _{r \rightarrow 0} \sup _{\substack{(X, t) \in K \\ 0<\rho \leq r}} \sigma(\Delta(X, t, \rho))^{-1} \int_{\Delta(X, t, \rho)}|f(Y, s)-a| d \sigma=0 .
$$

We are interested in understanding the implications of this condition on the regularity of the 'free boundary' $\partial \Omega$. To formulate our main theorem we need to properly introduce the notion of $\delta_{0}$-Reifenberg flat domains.

DEFINITION 3. If $\Omega$ is a connected open set in $\mathrm{R}^{n+1}$ then we say that $\partial \Omega$ separates $\mathrm{R}^{n+1}$ and is $\delta_{0}$-Reifenberg flat, $0<\delta_{0} \leq 1 / 10$, if given any $(X, t) \in$ $\partial \Omega, R>0$, there exists a $n$ dimensional plane $\hat{P}=\hat{P}(X, t, R)$, containing $(X, t)$ and a line parallel to the $t$ axis, having unit normal $\hat{n}=\hat{n}(X, t, R)$ such that

$$
\begin{aligned}
& \left\{(Y, s)+r \hat{n} \in C_{R}(X, t):(Y, s) \in \hat{P}, r>\delta_{0} R\right\} \subset \Omega, \\
& \left\{(Y, s)-r \hat{n} \in C_{R}(X, t):(Y, s) \in \hat{P}, r>\delta_{0} R\right\} \subset \mathrm{R}^{n+1} \backslash \Omega .
\end{aligned}
$$

For short we say that $\partial \Omega$ separates $\mathbf{R}^{n+1}$ when the last two conditions hold for some $\delta_{0}$.

Note that if $\partial \Omega$ separates $R^{n+1}$ in the sense of Definition 1 , then a line segment drawn parallel to $\hat{n}$ and with endpoints in each of the sets stated in the definition, also intersects $\partial \Omega$. We will often refer to $\Omega$ as being a $\delta_{0}$-Reifenberg flat domain if $\partial \Omega$ is $\delta_{0}$-Reifenberg flat. We pose one more definition.

Definition 4. Let $\Omega$ be a connected open set in $\mathrm{R}^{n+1},(X, t) \in \partial \Omega$, and $r>0$. We say that $C_{r}(X, t) \cap \partial \Omega$ is Reifenberg flat with vanishing constant in the parabolic sense, if for each $\epsilon>0$, there exists $\rho_{0}=\rho_{0}(\epsilon)>0$ with the following property. If $(\tilde{X}, \tilde{t}) \in C_{r}(X, t) \cap \partial \Omega$ and $0<\rho \leq \rho_{0}$, then there exists a plane $P^{\prime}(\tilde{X}, \tilde{t}, \rho)$ containing a line parallel to the $t$ axis such that the statement in Definition 3 holds with $R, \delta_{0}, \hat{P}$ replaced by $\rho, \epsilon, P^{\prime}$.

We are now ready to state our main theorem.

THEOREM 1. Assume that $\Omega$ is a two-sided NTA-domain with Ahlfors regular boundary. Let $\left(\hat{X}^{i}, \hat{t}^{i}\right) \in \Omega^{i}$, for $i \in\{1,2\}, \hat{t}^{2}<\hat{t}^{1}$ and assume that $\omega^{i}\left(\hat{X}^{i}, \hat{t}^{i}, \cdot\right)$, for $i \in\{1,2\}$, is absolutely continuous with respect to $\sigma$ on $\partial \Omega$ and that the Poisson kernels $k^{i}\left(\hat{X}^{i}, \hat{t}^{i}, \cdot\right)=d \omega^{i}\left(\hat{X}^{i}, \hat{t}^{i}, \cdot\right) / d \sigma$ are such that $\log k^{i}\left(\hat{X}^{i}, \hat{t}^{i}, \cdot\right) \in \operatorname{VMO}(d \sigma)$. Then $C_{r}(X, t) \cap \partial \Omega$ is Reifenberg flat with vanishing constant whenever $(X, t) \in \partial \Omega$ and $\hat{t}^{2}>t+4 r^{2}$.

To formulate an important consequence of Theorem 1, giving a theorem (Theorem 3) on free boundary regularity beyond the continuous threshold, we 
recall that through the works in [17], [9] it has become clear that from the perspective of parabolic singular integrals and caloric measure the parabolic analogue of the notion of Lipschitz domains, explored in elliptic partial differential equations, is graph domains $\Omega=\left\{(X, t) \in \mathrm{R}^{n+1}: x_{0}>\psi(x, t)\right\}$ where $\psi=\psi(x, t): \mathrm{R}^{n} \rightarrow \mathrm{R}$ has compact support and satisfies

$$
\begin{aligned}
& |\psi(x, t)-\psi(y, t)| \leq b_{1}|x-y|, \quad x, y \in \mathrm{R}^{n-1}, \quad t \in \mathrm{R}, \\
& D_{1 / 2}^{t} \psi \in B M O\left(\mathrm{R}^{n}\right), \quad\left\|D_{1 / 2}^{t} \psi\right\|_{*} \leq b_{2}<\infty .
\end{aligned}
$$

Here $D_{1 / 2}^{t} \psi(x, t)$ denotes the $1 / 2$ derivative in $t$ of $\psi(x, \cdot), x$ fixed. This half derivative in time can be defined by way of the Fourier transform or by

$$
D_{1 / 2}^{t} \psi(x, t) \equiv \hat{c} \int_{\mathrm{R}} \frac{\psi(x, s)-\psi(x, t)}{|s-t|^{3 / 2}} d s
$$

for properly chosen $\hat{c}$. $\|\cdot\|_{*}$ denotes the norm in parabolic $B M O\left(\mathrm{R}^{n}\right)$ (for a definition of this space see [11]). One can prove that the conditions in (2) and (3) imply that $\psi(x, t)$ is parabolically Lipschitz in the following sense,

$$
|\psi(x, t)-\psi(y, s)| \leq \beta\left(|x-y|+|t-s|^{1 / 2}\right) \quad x, y \in \mathbf{R}^{n} \quad t, s \in \mathbf{R} .
$$

Under the smoothness assumptions on $\psi$ stated in (2) and (3) it was proven in [17] that the parabolic Poisson kernel is in a certain $L^{p}$ reverse Hölder class for some $p>1$. In particular $\omega(\hat{X}, \hat{t}, \cdot)$ is an $A^{\infty}$ weight (with respect to $\sigma$ ). Finally we note that examples of [16] and [18] show that caloric and adjoint caloric measure need not be absolutely continuous with respect to the surface measure $\sigma$ in graph $\operatorname{Lip}(1,1 / 2)$ domain.

In [11] the parabolic Poisson kernel was analyzed in domains not locally given by graphs. In this situation the geometry was controlled by a certain geometric square function, the boundedness of which implied that on every scale the boundary contained 'big pieces of graph', graph with the regularity stated in (2) and (3) (see [10]). A fundamental assumption in [11] is that $\partial \Omega$ is $\delta_{0}$-Reifenberg flat and satisfies a $(M, R)$ Ahlfors condition but to properly formulate the result in [11] we need to introduce some more notation and concepts.

Let

$$
d\left(F_{1}, F_{2}\right)=\inf \left\{|X-Y|+|s-t|^{1 / 2}:(X, t) \in F_{1},(Y, s) \in F_{2}\right\}
$$

denote the parabolic distance between the sets $F_{1}, F_{2}$ and for $\Omega$ (such that $\partial \Omega$ separates $\mathrm{R}^{n+1}$ and satisfies a $(M, R)$ Ahlfors condition) we set

$$
\gamma(Z, \tau, r)=\inf _{P}\left[r^{-n-3} \int_{\partial \Omega \cap C_{r}(Z, \tau)} d(\{(Y, s)\}, P)^{2} d \sigma(Y, s)\right] .
$$


Here the infimum is taken over all $n$ dimensional planes $P$ containing a line parallel to the $t$ axis. Let

$$
d \nu(Z, \tau, r)=\gamma(Z, \tau, r) d \sigma(Z, \tau) r^{-1} d r .
$$

We say that $v$ is a Carleson measure on $\left[\partial \Omega \cap C_{R}(Y, s)\right] \times(0, R)$ if there exists $M_{1}<\infty$ such that whenever $(X, t) \in \partial \Omega$ and $C_{\rho}(X, t) \subset C_{R}(Y, s)$, we have

$$
v\left(\left[C_{\rho}(X, t) \cap \partial \Omega\right] \times(0, \rho)\right) \leq M_{1} \rho^{n+1} .
$$

The smallest such $M_{1}$ is called the Carleson norm of $v$ on $\left[\partial \Omega \cap C_{R}(Y, s)\right] \times$ $(0, R)$ and we write $\|v\|_{+}$for the Carleson norm of $v$ if the inequality in (4) holds for all $\rho>0$. The following two definitions can be found in [10] and [11].

DEFINITION 5. $\partial \Omega$ is said to be uniformly rectifiable (in the parabolic sense) if $\|v\|_{+}<\infty$ and if (4) and (1) hold for all $\rho>0$ and $R>0$ respectively. If furthermore $\partial \Omega$ separates $R^{n+1}$ and is uniformly rectifiable, then $\Omega$ is called a parabolic regular domain.

DEFINITION 6. $\Omega$ is called a chord arc domain with vanishing constant if $\Omega$ is a parabolic regular domain and

$$
\sup _{\substack{(X, t) \in \partial \Omega \\ 0<\rho \leq r}}\left[\rho^{-(n+1)} v\left(\left[C_{\rho}(X, t) \cap \partial \Omega\right] \times(0, \rho)\right)\right] \rightarrow 0 \quad \text { as } \quad r \rightarrow 0 .
$$

To formulate the result in [11] which is relevant to the discussions in this paper we for $(X, t) \in \partial \Omega$, and $r, \rho>0$ define $\Delta(X, t, r, \rho)=\{(Y, s) \in$ $\left.\partial \Omega:|Y-X|<r,|s-t|<\rho^{2}\right\}$. In this notation $\Delta(X, t, r)=\Delta(X, t, r, r)$. We say that $\omega(\hat{X}, \hat{t}, \cdot)$ is asymptotically optimal doubling if whenever $K \subset$ $\partial \Omega \cap\{(Y, s): s<\hat{t}\}$ is compact and $0<\tau_{1}, \tau_{2}<1$, we have

$$
\begin{aligned}
\lim _{r \rightarrow 0} \sup _{(X, t) \in K} \frac{\omega\left(\Delta\left(X, t, \tau_{1} r, \tau_{2} r\right)\right)}{\omega(\Delta(X, t, r))} & =\lim _{r \rightarrow 0} \inf _{(X, t) \in K} \frac{\omega\left(\Delta\left(X, t, \tau_{1} r, \tau_{2} r\right)\right)}{\omega(\Delta(X, t, r))} \\
& =\tau_{1}^{n-1} \tau_{2}^{2} .
\end{aligned}
$$

In [11] it is proven that if $\Omega$ is a parabolic regular domain with Reifenberg constant $\delta_{0}=\delta_{0}\left(M,\|v\|_{+}\right)$, sufficiently small, then $\omega$ is an $A^{\infty}$ weight. Also if $\Omega$ is a chord arc domain with vanishing constant and $k(\hat{X}, \hat{t}, \cdot)=d \omega(\hat{X}, \hat{t}, \cdot) / d \sigma$, then $\log k(\hat{X}, \hat{t}, \cdot) \in \operatorname{VMO}(d \sigma)$. Furthermore in [11] the following theorem is proven.

THEOREM 2. Let $\Omega$ be a parabolic regular domain and put $k(\hat{X}, \hat{t}, \cdot)=$ $d \omega(\hat{X}, \hat{t}, \cdot) / d \sigma$. If $\omega(\hat{X}, \hat{t}, \cdot)$ is asymptotically optimal doubling, $\log k(\hat{X}, \hat{t}, \cdot)$ 
$\in \operatorname{VMO}(d \sigma)$ and $\|v\|_{+}$is small enough then (5) holds with $\partial \Omega$ replaced by any compact subset, $F \subset \partial \Omega \cap\{(Y, s): s<\hat{t}\}$.

Combining Theorem 1, Theorem 2 and Proposition 5.1 in [11] we can state the following theorem.

THEOREM 3. Assume that $\Omega$ is a two-sided NTA-domain with Ahlfors regular boundary and that $\Omega$ is a parabolic regular domain. Let $\left(\hat{X}^{i}, \hat{t}^{i}\right) \in \Omega^{i}$, for $i \in\{1,2\}, \hat{t}^{2}<\hat{t}^{1}$ and assume that the Poisson kernels $k^{i}\left(\hat{X}^{i}, \hat{t}^{i}, \cdot\right)=$ $d \omega^{i}\left(\hat{X}^{i}, \hat{t}^{i}, \cdot\right) / d \sigma$ are such that $\log k^{i}\left(\hat{X}^{i}, \hat{t}^{i}, \cdot\right) \in \operatorname{VMO}(d \sigma)$ for $i \in\{1,2\}$. If $\|v\|_{+}$is small enough and $F$ is a compact subset of $\partial \Omega \cap\{(Y, s): s<\hat{t}\}$, then (5) holds with $\partial \Omega$ replaced by $F$.

The theorem states that the conditions $\log k^{1}\left(\hat{X}^{1}, \hat{t}^{1}, \cdot\right) \in \operatorname{VMO}(d \sigma)$ and $\log k^{2}\left(\hat{X}^{2}, \hat{t}^{2}, \cdot\right) \in \operatorname{VMO}(d \sigma)$ can serve, from the perspective of Theorem 3 , as a replacement for the condition that $\omega(\hat{X}, \hat{t}, \cdot)$ is asymptotically optimal doubling.

Concerning related elliptic free boundary problems a classical result of AltCaffarelli states (for the definition of all the concepts we refer to [1] and [14]) that if $\Omega \subset \mathrm{R}^{n}$ is $\delta$-Reifenberg flat with an Ahlfors regular boundary and if $\log k \in C^{0, \beta}(\partial \Omega)$ for some $\beta \in(0,1)$, then $\Omega$ is a $C^{1, \alpha}$-domain for some $\alpha \in(0,1)$ which depends on $\beta$ and $n$. Similar problems was studied by Kenig and Toro, in the setting of domains not locally given by graphs, and in [14] (see also [12] and [13]) the authors prove the following theorem which is the analogue of the result of [1] assuming vanishing oscillation of the logarithm of the Poisson kernel in an integral sense $(\operatorname{VMO}(d \sigma))$ instead of in the classical pointwise sense.

THeOREm 4. Assume that $\Omega \subset \mathrm{R}^{n}$ is $\delta_{0}$-Reifenberg flat for some small enough $\delta_{0}>0$ and assume that $\partial \Omega$ is Ahlfors regular. If $\log k \in \mathrm{VMO}(d \sigma)$ then $\Omega$ is a chord arc domain with vanishing constant, i.e., the measure theoretical normal $\vec{n}$ is in $\operatorname{VMO}(d \sigma)$.

Furthermore in [15], Kenig and Toro consider the elliptic version of the two-phase problem we consider in Theorem 1 and Theorem 3. In particular assuming that $\Omega \subset \mathrm{R}^{n}$ is a two-sided chord arc domain (meaning that $\Omega^{1}$ and $\Omega^{2}$ are NTA-domains and that $\partial \Omega$ is Ahlfors) they prove ([15, Corollary 5.2]) that if $\log k^{1} \in \operatorname{VMO}(d \sigma)$ and $\log k^{2} \in \operatorname{VMO}(d \sigma)$ then firstly $\partial \Omega$ is Reifenberg flat with vanishing constant and secondly $\Omega$ is a chord arc domain with vanishing constant, i.e., the measure theoretical normal $\vec{n}$ is in $\operatorname{VMO}(d \sigma)$.

Our long term goal is to establish the parabolic version of Theorem 4 (which can be refered to as a one-phase version of the two-phase problem we consider in Theorem 1 and Theorem 3) but we note that the proof in [14] uses the important and deep result of [1] for elliptic partial differential equations. The potential 
generalization of these results to the heat equation is currently unknown and these 'free boundary' type problems do in fact appear harder in the caloric case. By imposing the two-phase condition $\log k^{1}\left(\hat{X}^{1}, \hat{t}^{1}, \cdot\right) \in \operatorname{VMO}(d \sigma)$ and $\log k^{2}\left(\hat{X}^{2}, \hat{t}^{2}, \cdot\right) \in \operatorname{VMO}(d \sigma)$ we do not need a caloric version of the result of [1] and this is one of the main reasons we are able to make progress. Though similar problems have been considered in [2], [3] under much stronger assumptions we want to emphasize that our main results, Theorem 1 and Theorem 3, are completely new and that we are not aware of any competing results of this type in the parabolic setting. Finally we note that in [20] a similar but different problem concerning caloric measure and Reifenberg flatness is studied.

The rest of the paper is organized as follows. In section 2 we in section list some basic estimates for solutions to the heat - adjoint heat equation in parabolic NTA-domains. These estimates are then complemented by an estimate based on an exploration of the condition $\log k(\hat{X}, \hat{t}, \cdot) \in \operatorname{VMO}(d \sigma)$. In section 2.2 we clarify the notion of Green function with pole at infinity and the associated caloric measure. In section 3, which is at the heart of the matter, our regularity assumptions on the kernel $k^{i}\left(\hat{X}^{i}, \hat{t}^{i}, \cdot\right)$ is explored in a blow-up argument. In the limit we encounter a problem of classification of what we refer to as global solutions to a specific two-phase free boundary problem. The section ends with a theorem giving us the appropriate classification and finally it is shown that Theorem 1 is a consequence of that classification theorem.

\section{Estimates of caloric functions in parabolic NTA-domains}

Recall from [17, ch. 3, section 6] that $\Omega \subset \mathrm{R}^{n+1}$ is an unbounded parabolic nontangentially accessible domain, NTA-domain for short, if $\partial \Omega$ separates $\mathrm{R}^{n+1}$ and if the following conditions are satisfied for some $\lambda, \gamma \geq 100$. Given $(X, t) \in \partial \Omega$ and $r>0$ there exist

$$
\begin{aligned}
& \underline{A}_{r}^{1}(X, t)=\left(U_{1}(X, t), t_{1}(X, t)\right)=\left(U_{1}, t_{1}\right) \in \Omega \cap C_{r}(X, t), \\
& \bar{A}_{r}^{1}(X, t)=\left(U_{2}(X, t), t_{2}(X, t)\right)=\left(U_{2}, t_{2}\right) \in \Omega \cap C_{r}(X, t), \\
& \underline{A}_{r}^{2}(X, t)=\left(N_{1}(X, t), \tau_{1}(X, t)\right)=\left(N_{1}, \tau_{1}\right) \in\left(\mathrm{R}^{n+1} \backslash \bar{\Omega}\right) \cap C_{r}(X, t), \\
& \bar{A}_{r}^{2}(X, t)=\left(N_{2}(X, t), \tau_{2}(X, t)\right)=\left(N_{2}, \tau_{2}\right) \in\left(\mathrm{R}^{n+1} \backslash \bar{\Omega}\right) \cap C_{r}(X, t),
\end{aligned}
$$

such that

$$
\begin{aligned}
& \lambda^{-1} r^{2} \leq \min \left(t_{2}-t, t-t_{1}\right) \leq \lambda r^{2}, \\
& \lambda^{-1} r^{2} \leq \min \left(\tau_{2}-t, t-\tau_{1}\right) \leq \lambda r^{2}, \\
& r / \lambda \leq \min \left[d\left(\left\{\left(N_{i}, \tau_{i}\right)\right\}, \partial \Omega\right), d\left(\left\{\left(U_{i}, t_{i}\right)\right\}, \partial \Omega\right)\right] .
\end{aligned}
$$


Here $d(\cdot, \cdot)$ denotes the parabolic distance defined in the introduction. As in [JK1] we refer to these conditions as the corkscrew condition. Next suppose $\left(U_{i}, s_{i}\right) \in \Omega, i=1,2$, with $\left(s_{2}-s_{1}\right)^{\frac{1}{2}}>\gamma^{-1} d\left(\left\{\left(U_{1}, s_{1}\right)\right\},\left\{\left(U_{2}, s_{2}\right)\right\}\right)$. We say as in [JK1] that $\left\{C_{r_{i}}\left(X_{i}, t_{i}\right)\right\}_{1}^{l}$ is a Harnack chain from $\left(U_{1}, s_{1}\right)$ to $\left(U_{2}, s_{2}\right)$ with constant $\gamma$ provided there exists $c(\gamma) \geq 1$ such that

- $\left(U_{1}, s_{1}\right) \in C_{r_{1}}\left(X_{1}, t_{1}\right),\left(U_{2}, s_{2}\right) \in C_{r_{l}}\left(X_{l}, t_{l}\right)$, and $C_{r_{i+1}}\left(X_{i+1}, t_{i+1}\right) \cap$ $C_{r_{i}}\left(X_{i}, t_{i}\right) \neq \emptyset$ for $i=1,2, \ldots, l-1$,

- $c(\gamma)^{-1} d\left(\left\{\left(X_{i}, t_{i}\right)\right\}, \partial \Omega\right) \leq r_{i} \leq c(\gamma) d\left(\left\{\left(X_{i}, t_{i}\right)\right\}, \partial \Omega\right)$, when $i=$ $1,2, \ldots, l$,

- $t_{i+1}-t_{i} \geq c(\gamma)^{-1} r_{i}^{2}$, for $i=1,2, \ldots, l$,

- $l \leq c(\gamma) \log \left(2+\frac{d\left(\left\{\left(U_{1}, s_{1}\right)\right\},\left\{\left(U_{2}, s_{2}\right)\right\}\right)}{\min \left[d\left(\left\{\left(U_{1}, s_{1}\right)\right\}, \partial \Omega\right), d\left(\left\{\left(U_{2}, s_{2}\right)\right\}, \partial \Omega\right)\right]}\right)$.

$l$ is refered to as the length of the Harnack chain. For $(X, t) \in \partial \Omega$ and $r>0$ we define the following points located in $\Omega$,

$$
\underline{A}_{r}(X, t)=\underline{A}_{r}^{1}(X, t), \quad \bar{A}_{r}(X, t)=\bar{A}_{r}^{1}(X, t) .
$$

By $A_{r}(X, t)$ we will denote a point in $\Omega$ such that if $A_{r}(X, t)=\left(A_{r}^{x}(X, t)\right.$, $\left.A_{r}^{t}(X, t)\right)$ then $A_{r}^{t}(X, t)=t$ and $d\left(A_{r}(X, t), \partial \Omega\right) \sim r \sim d\left(A_{r}(X, t),(X, t)\right)$. The existence of such points is a consequence of the fact that $\Omega$ is a parabolic NTA-domain and we will make use of these points throughout the section. If $(Y, s) \in \Omega$ then we let $\delta(Y, s)$ denote the parabolic distance from $(Y, s)$ to $\partial \Omega$.

\subsection{Basic estimates}

In this section we state some basic estimates for certain solutions to the heat and adjoint heat equation in parabolic NTA-domains. An outline of the proofs of these lemmas valid in the current situation can be found in [17, ch. 3, section 6] and [11]. Apart from these references many of the relevant ideas used in the proofs can also be found in [5], [6] and [19]. In particular, in [19] all relevant estimates are stated and proved, in $\operatorname{Lip}(1,1 / 2)$ domains, in the general setting of second order parabolic equations in divergence form.

Note that the characteristics of a parabolic NTA-domain is described by the parameters $\lambda$ and $\gamma$ and hence basically all constants appearing below will depend on these two parameters. I.e., below $c=c(\lambda, \gamma)$ but the constants often also depend on other parameters and we will not always indicate the dependence on $\lambda$ and $\gamma$.

We start by a lemma on Hölder decay at the boundary of non-negative solutions vanishing on the boundary. The lemma is proved using standard 
comparison arguments and the fact that the complement of $\Omega$ is uniformly 'fat'.

Lemma 5. Let $\Omega \subset \mathrm{R}^{n+1}$ be a parabolic NTA-domain with parameters $\lambda$ and $\gamma$. Let $(X, t) \in \partial \Omega$ and suppose that $u$ is a non-negative solution to either the heat or the adjoint heat equation in $\Omega \cap C_{2 r}(X, t)$ which vanishes continuously on $\partial \Omega \cap C_{2 r}(X, t)$. Then there exists $\alpha=\alpha(\lambda, \gamma), 0<\alpha<\frac{1}{2}$, and $c=c(\lambda, \gamma) \geq 1$ such that whenever $(Y, s) \in \Omega \cap C_{r}(X, t)$

$$
u(Y, s) \leq c\left[\frac{d(\{(Y, s)\},\{(X, t)\})}{r}\right]^{\alpha} \sup _{(Z, \tau) \in \Omega \cap C_{r}(X, t)} u(Z, \tau) .
$$

The next lemma is a standard Carleson type lemma.

Lemma 6. Let $u, \Omega$ and $(X, t)$ be as in the previous lemma. If $(Y, s) \in$ $\Omega \cap C_{r / 2}(X, t)$, then

$$
u(Y, s) \leq c u\left(\bar{A}_{r}(X, t)\right)
$$

when $u$ is a solution to the heat equation while

$$
u(Y, s) \leq c u\left(\underline{A}_{r}(X, t)\right)
$$

when $u$ is a solution to the adjoint heat equation in $C_{2 r}(X, t) \cap \Omega$.

Given $(Y, s) \in \Omega$, let $G(\cdot, Y, s)$ denote Green's function for the heat equation in $\Omega$ with pole at $(Y, s)$. That is

$$
\begin{aligned}
\frac{\partial}{\partial t} G(X, t, Y, s)-\Delta & G(X, t, Y, s) \\
& =\delta((X, t)-(Y, s)) \quad \text { in } \Omega \text { and } G \equiv 0 \text { on } \partial \Omega .
\end{aligned}
$$

Here $\delta$ denotes the Dirac delta function and $\Delta$ is the Laplacian in $X$. We note that $G(Y, s, \cdot)$ is the Green's function for the adjoint heat equation with pole at $(Y, s) \in \Omega$ (i.e. $-\frac{\partial}{\partial t} G(Y, s, \cdot)-\Delta G(Y, s, \cdot)=\delta(\cdot-(Y, s))$. Let $\omega, \hat{\omega}$ be the corresponding caloric and adjoint caloric measures for the heat - adjoint heat equation in $\Omega$. We note that $\omega(Y, s, \cdot), \hat{\omega}(Y, s, \cdot)$ are the Riesz measures associated with $G(Y, s, \cdot), G(\cdot, Y, s)$ by way of the Riesz representation theorem for sub caloric - adjoint caloric functions in $\mathrm{R}^{n+1} \backslash\{(Y, s)\}$ (see [4]). From this theorem we have that

$$
\int_{\partial \Omega} \phi d \omega(Y, s, \cdot)=\int_{\Omega} G(Y, s, \cdot)\left(\Delta \phi-\frac{\partial \phi}{\partial \tau}\right) d Z d \tau
$$

for all $\phi \in C_{0}^{\infty}\left(\mathrm{R}^{n+1} \backslash\{(Y, s)\}\right)$. A similar formula holds for $\hat{\omega}$. Estimates for caloric - adjoint caloric measure in terms of the Green's function and vice versa are given by the following lemma. The proof follows by standard arguments. 
Lemma 7. Let $\Omega$ and $(X, t)$ be as in the previous lemma. Let $A \geq 100$ and assume that $(Y, s) \in \Omega$ with $|Y-X|^{2} \leq A|s-t|$ and $|s-t| \geq 4 r^{2}$. There exists $c=c(A) \geq 1$ such that if $s>t$, then

$$
c^{-1} r^{n} G\left(Y, s, \bar{A}_{r}(X, t)\right) \leq \omega(Y, s, \Delta(X, t, r / 2)) \leq c r^{n} G\left(Y, s, \underline{A}_{r}(X, t)\right)
$$

while if $s<t$,

$$
c^{-1} r^{n} G\left(\underline{A}_{r}(X, t), Y, s\right) \leq \hat{\omega}(Y, s, \Delta(X, t, r / 2)) \leq c r^{n} G\left(\bar{A}_{r}(X, t), Y, s\right) .
$$

Next we have the following backward Harnack inequality.

Lemma 8. Let $\Omega$ and $(X, t)$ be as in the previous lemma. Let $A \geq 100$ and assume that $|Y-X|^{2} \leq A|s-t|$ and $|s-t| \geq 5 r^{2}$. There exists $c=c(A) \geq 1$ such that

$$
G\left(Y, s, \underline{A}_{r}(X, t)\right) \leq c G\left(Y, s, \bar{A}_{r}(X, t)\right)
$$

when $s>t$ while if $s<t$, then

$$
G\left(\underline{A}_{r}(X, t), Y, s\right) \leq c G\left(\bar{A}_{r}(X, t), Y, s\right) .
$$

Combining the previous two lemmas the doubling property of caloric adjoint caloric measure can be proven.

Lemma 9. Let $\Omega,(X, t),(Y, s)$ and $A$ be as in the previous lemma. Then

$$
\omega^{*}(Y, s, \Delta(X, t, r)) \leq c(A) \omega^{*}(Y, s, \Delta(X, t, r / 2))
$$

where $\omega^{*}=\omega$ when $s>t$ while $\omega^{*}=\hat{\omega}$ when $s<t$.

Let $(X, t) \in \partial \Omega, \rho>0$ and $R>0 . u>0$ is said to satisfy a strong Harnack inequality in $C_{R}(X, t) \cap \Omega$ provided that $u$ is a solution to either the heat or adjoint heat equation in $C_{R}(X, t) \cap \Omega$ and

$u(\hat{X}, \hat{t}) \leq \tilde{\lambda} u(\tilde{X}, \tilde{t})$ whenever $(\hat{X}, \hat{t}),(\tilde{X}, \tilde{t}) \in C_{\rho}(Z, \tau)$

$$
\text { and } C_{2 \rho}(Z, \tau) \subset C_{R}(X, t) \cap \Omega \text {. }
$$

Here $\tilde{\lambda}, 1 \leq \tilde{\lambda}<\infty$, is independent of $C_{2 \rho}(Z, \tau) \subset C_{R}(X, t) \cap \Omega$. For $(X, t)$, $\rho$ as above and $A>0$ we define

$$
\begin{aligned}
& \Gamma_{A}^{+}(X, t, \rho)=\Omega \cap\left\{(Y, s):|Y-X|^{2} \leq A|s-t|,|s-t| \geq 5 \rho^{2}, s>t\right\}, \\
& \Gamma_{A}^{-}(X, t, \rho)=\Omega \cap\left\{(Y, s):|Y-X|^{2} \leq A|s-t|,|s-t| \geq 5 \rho^{2}, s<t\right\} .
\end{aligned}
$$

Using Lemma 7, Lemma 8 and Lemma 9 one can prove that if $(Y, s) \in$ $\Gamma_{A}^{+}(X, t, R)$ then $G(Y, s, \cdot)$ satisfies a strong Harnack inequality in $C_{R}(X, t) \cap$ 
$\Omega$ while if $(Y, s) \in \Gamma_{A}^{-}(X, t, R)$ then $G(\cdot, Y, s)$ satisfies a strong Harnack inequality in $C_{R}(X, t) \cap \Omega$. Moreover, $\tilde{\lambda}$ depends only on $A$ once the NTAconstants $\lambda$ and $\gamma$ have been chosen. Using the notion of strong Harnack inequality the following two comparison lemmas can be proven.

Lemma 10. Let $u, v>0$ be continuous in $\bar{C}_{2 r}(X, t) \cap \bar{\Omega}, u=v=0$ on $\Delta(X, t, 2 r)$ and assume that $u$ and $v$ both are solutions either to the heat or the adjoint heat equation in $C_{2 r}(X, t) \cap \Omega$. If $u, v$ satisfy a strong Harnack inequality in $C_{2 r}(X, t) \cap \Omega$ for some $\tilde{\lambda} \geq 1$, then

$$
\frac{u(Y, s)}{v(Y, s)} \leq c(\tilde{\lambda}) \frac{u(\hat{U})}{v(\hat{U})} \quad \text { in } \quad C_{r / 2}(X, t) \cap \bar{\Omega} .
$$

Here $\hat{U}=\bar{A}_{r}(X, t)$ when $u, v$ are solutions to the heat equation while $\hat{U}=$ $\underline{A}_{r}(X, t)$ when $u, v$ are solutions to the adjoint heat equation in $\Omega \cap C_{2 r}(X, t)$.

LEMMA 11. Under the same hypotheses as in Lemma 10 there exists $\tilde{\gamma}=$ $\tilde{\gamma}(\tilde{\lambda}), 0<\tilde{\gamma} \leq 1 / 2$, and $c=c(\tilde{\lambda}) \geq 1$, such that whenever $0<\rho \leq r / 2$ then

$$
\left|\frac{u(Z, \tau) v(Y, s)}{v(Z, \tau) u(Y, s)}-1\right| \leq c(\rho / r)^{\tilde{\gamma}} \quad \text { for } \quad(Z, \tau),(Y, s) \in C_{\rho}(X, t) \cap \Omega .
$$

In the following we will assume that $\Omega \subset \mathrm{R}^{n+1}$ is a parabolic NTA-domain with Ahlfors regular boundary. Let $(\hat{X}, \hat{t}) \in \Omega$ and define $\omega(\hat{X}, \hat{t}, \cdot)$ to be the caloric measure defined w.r.t. $\Omega$. In the following we will assume that $\omega(\hat{X}, \hat{t}, \cdot)$ is absolutely continuous with respect to the surface measure $\sigma$. We define the Poisson kernel as $k(\hat{X}, \hat{t}, \cdot)=d \omega(\hat{X}, \hat{t}, \cdot) / d \sigma$ and we will assume that $\log k(\hat{X}, \hat{t}, \cdot) \in \operatorname{VMO}(d \sigma)$. We start by exploring the information contained in this condition.

LEMMA 12. Let $\Omega \subset \mathrm{R}^{n+1}$ be a parabolic NTA-domain with Ahlfors regular boundary. Assume that $\omega(\hat{X}, \hat{t}, \cdot)$ is absolutely continuous with respect to the surface measure $\sigma$ and that the Poisson kernel $k(\hat{X}, \hat{t}, \cdot)=d \omega(\hat{X}, \hat{t}, \cdot) / d \sigma$ is such that

$$
\log k(\hat{X}, \hat{t}, \cdot) \in \operatorname{VMO}(d \sigma) .
$$

Then given $\epsilon \in(0,1)$ there exists a constant $C=C(\epsilon, n, \Omega, A)$ such that for all $(X, t) \in \partial \Omega, r<r_{0},|X-\hat{X}|^{2} \leq A(\hat{t}-t)$ for some $A \geq 2, \hat{t}-t \geq 8 r^{2}$ and $E \subset \Delta(X, t, r)$,

$$
C^{-1}\left(\frac{\sigma(E)}{\sigma(\Delta(X, t, r))}\right)^{1+\epsilon} \leq \frac{\omega(\hat{X}, \hat{t}, E)}{\omega(\hat{X}, \hat{t}, \Delta(X, t, r))} \leq C\left(\frac{\sigma(E)}{\sigma(\Delta(X, t, r))}\right)^{1-\epsilon} .
$$


Proof. Let $p=\epsilon^{-1}$ and let $E \subset \Delta(X, t, r)$ and $\Delta(X, t, r)$ be as in the statement of the lemma. Note that the restrictions on the points $(X, t)$ and $(\hat{X}, \hat{t})$ imply control of the doubling constants of the caloric measure. Also note that the information on $\Omega$ entering into the constant $C=C(\epsilon, n, \Omega, A)$ is simply the NTA-parameters and the constants appearing in the Ahlfors condition. In the following we write $k(Y, s)=k(\hat{X}, \hat{t}, Y, s), \omega(E)=\omega(\hat{X}, \hat{t}, E)$. By Cauchy-Schwarz we have

$$
\omega(E)=\int_{E} k(Y, s) d \sigma(Y, s) \leq\left(\int_{\Delta(X, t, r)}[k(Y, s)]^{p} d \sigma(Y, s)\right)^{1 / p} \sigma(E)^{1-1 / p} .
$$

As a consequence of the John-Nirenberg inequality, as $\partial \Omega$ satisfies a condition of Ahlfors type w.r.t. the surface measure, (see for instance the discussion above Theorem 2.1 in [14] as well as [7], [8]) the assumption $\log k \in \operatorname{VMO}(d \sigma)$ implies that if $1<p<\infty$ the following reverse type Hölder inequalities are true,

$$
\begin{aligned}
& \left(\int_{\Delta(X, t, r)}[k(Y, s)]^{p} d \sigma(Y, s)\right)^{1 / p} \\
& \leq C[\sigma(\Delta(X, t, r))]^{1 / p-1} \int_{\Delta(X, t, r)} k(Y, s) d \sigma(Y, s), \\
& \left(\int_{\Delta(X, t, r)}[k(Y, s)]^{-p} d \sigma(Y, s)\right)^{1 / p} \\
& \leq C[\sigma(\Delta(X, t, r))]^{1 / p-1} \int_{\Delta(X, t, r)}[k(Y, s)]^{-1} d \sigma(Y, s) .
\end{aligned}
$$

Combining we get

$\omega(E)=\int_{E} k(Y, s) d \sigma(Y, s) \leq C \sigma(E)^{1-1 / p} \sigma(\Delta(X, t, r))^{1 / p-1} \omega(\Delta(X, t, r))$.

This completes the proof in one direction. The second direction is proved similarly.

\subsection{The Green function and caloric measure at infinity}

In this section we will clarify the notion of Green function with pole at infinity and the associated caloric measure.

Lemma 13. Let $\Omega$ be a parabolic NTA-domain. Then there exists a unique function $u$ (unique modulo a constant) such that $u$ is a non-negative solution to the adjoint heat in $\Omega$ and such that $u$ vanishes continuously on $\partial \Omega$. 
In fact a similar result holds for the heat equation. The function $u$, in the statement of the lemma, should be refered to as the Green function with pole at +infinity. By + we refer to the 'infinity' in the positive direction of time.

Proof. There are two steps in the proof, the uniqueness and the existence. We start by proving the existence. We let $(X, t) \in \partial \Omega$ and let $R>0$ be a large positive number. Assume that $(\hat{X}, \hat{t}) \in \Gamma_{A}^{+}(X, t, 100 R)$ and let $K \subset \mathrm{R}^{n+1}$ be a fixed compact set. Assume that $R$ is so large that $K \cap \Omega \subset C_{R}(X, t)$. Using Lemma 6 , the fact that if $(\hat{X}, \hat{t}) \in \Gamma_{A}^{+}(X, t, 100 R)$ then $G(\hat{X}, \hat{t}, \cdot)$ satisfies a strong Harnack inequality in $C_{R}(X, t) \cap \Omega$ and Lemma 10 it follows that if $(Z, \tau) \in K \cap \Omega$, then

$$
G(\hat{X}, \hat{t}, Z, \tau) \leq C_{K, n, A} G\left(\hat{X}, \hat{t}, A_{1}(X, t)\right) .
$$

In particular this implies that if $(\hat{X}, \hat{t}) \in \Gamma_{A}^{+}(X, t, 100 R)$ then

$$
\sup _{(Z, \tau) \in K \cap \Omega} \frac{G(\hat{X}, \hat{t}, Z, \tau)}{G\left(\hat{X}, \hat{t}, A_{1}(X, t)\right)} \leq C_{K, n, A} .
$$

Let $\left(\hat{X}_{j}, \hat{t}_{j}\right) \in \Gamma_{A}^{+}\left(X, t, 2^{j} R\right)$ for $j=1,2, \ldots$ and define for $(Z, \tau) \in$ $C_{R}(X, t) \cap \Omega$

$$
u_{j}(Z, \tau)=\frac{G\left(\hat{X}_{j}, \hat{t}_{j}, Z, \tau\right)}{G\left(\hat{X}_{j}, \hat{t}_{j}, A_{1}(X, t)\right)} .
$$

Then $\left\{u_{j}\right\}$ is a set of positive adjoint caloric functions in $C_{R}(X, t) \cap \Omega$ vanishing on $\partial \Omega$. Furthermore, we can assume that $\left\{u_{j}\right\}$ is a uniformly bounded set of functions on $\Omega \cap C_{R}(X, t)$. By the Arzela-Ascoli theorem there exists a subsequence $\left\{\tilde{j}_{k}\right\}$ such that $\left\{u_{\tilde{j}_{k}}\right\}$ converges to a non-negative solution $\tilde{u}=\tilde{u}_{R}$ to the adjoint heat equation in $\Omega \cap C_{R}(X, t)$. If we choose a sequence of numbers $R_{i}$ such that $R_{i} \rightarrow \infty$ and pick a diagonal subsequence we can conclude that there exists a subsequence $\left\{j_{k}\right\}$ such that $\left\{u_{j_{k}}\right\}$ converges to a non-negative solution $u_{\infty}$ to the adjoint heat equation in $\Omega$, uniformly on compact sets of $\Omega$. Furthermore, $u_{\infty}$ vanishes continuously on $\partial \Omega$ and $u_{\infty}\left(A_{1}(X, t)\right)=1$.

Left is to prove uniqueness. Let $u$ and $v$ be two function fulfilling the statement of the lemma and assume that $u\left(A_{1}(X, t)\right)=v\left(A_{1}(X, t)\right)$ for some point $(X, t) \in \partial \Omega$. Under these assumptions we want to prove that $u \equiv v$. Let $\rho$ and $R$ be fixed numbers such that $0<\rho \leq R / 2$. Using the same argument as in the proof of Lemma 8 (see the proof of Lemma 3.11 in [11]) one can prove that $u$ and $v$ satisfy a strong Harnack inequality in $C_{R}(X, t) \cap$ $\Omega$ with a constant $\tilde{\lambda}$ which only depends on the characteristics of the NTAdomain and the dimension $n$. Using Lemma 11 we therefore get that whenever 
$(Z, \tau),(Y, s) \in C_{\rho}(X, t) \cap \Omega$ then

$$
\left|\frac{u(Z, \tau) v(Y, s)}{v(Z, \tau) u(Y, s)}-1\right| \leq c(\rho / R)^{\gamma} .
$$

Hence if we put $(Y, s)=A_{1}(X, t)$ then

$$
\left|\frac{u(Z, \tau)}{v(Z, \tau)}-1\right| \leq c(\rho / R)^{\gamma}
$$

whenever $(Z, \tau) \in C_{\rho}(X, t) \cap \Omega$. Letting $R \rightarrow \infty$ completes the proof.

Lemma 14. Let $\Omega$ be a parabolic NTA-domain and let $(X, t) \in \partial \Omega$. Then there exists a unique doubling Radon measure $\omega$ such that $\omega(\Delta(X, t, 1))=1$ and a non-negative solution $u$ to the adjoint heat in $\Omega$ vanishing continuously on $\partial \Omega$ such that for all $\phi \in C_{0}^{\infty}\left(\mathrm{R}^{n+1}\right)$

$$
\int_{\partial \Omega} \phi(Y, s) d \omega(Y, s)=\int_{\Omega} u(Y, s)\left(\Delta-\partial_{s}\right) \phi(Y, s) d Y d s .
$$

$\omega$ is refered to as the caloric measure for $\Omega$ at + infinity and normalized at $(X, t)$.

Proof. Again there are two steps in the proof, the uniqueness and the existence. In this case we start by proving the uniqueness. I.e., we assume that there exist two measures $\omega_{1}$ and $\omega_{2}$ as in the statement of the lemma and such that $\omega_{1}(\Delta(X, t, 1))=\omega_{2}(\Delta(X, t, 1))=1$ for a point $(X, t) \in \partial \Omega$. We want to prove that $\omega_{1} \equiv \omega_{2}$. Let $u_{1}$ and $u_{2}$ be related to $\omega_{1}$ respectively $\omega_{2}$ according to the statement of the lemma. Using Lemma 13 we can conclude that there exist constants $\alpha_{1}$ and $\alpha_{2}$ as well as a function $u$ such that $u_{i}=\alpha_{i} u$. Here $u$ is a non-negative solution to the adjoint heat in $\Omega$ such that $u$ vanishes continuously on $\partial \Omega$. I.e., for all $\phi \in C_{0}^{\infty}\left(\mathrm{R}^{n+1}\right)$

$$
\int_{\partial \Omega} \phi(Y, s) d \omega_{i}(Y, s)=\alpha_{i} \int_{\Omega} u(Y, s)\left(\Delta-\partial_{s}\right) \phi(Y, s) d Y d s .
$$

From this we can we conclude that

$$
\alpha_{1}^{-1} \int_{\partial \Omega} \phi(Y, s) d \omega_{1}(Y, s)=\alpha_{2}^{-1} \int_{\partial \Omega} \phi(Y, s) d \omega_{2}(Y, s) .
$$

Choosing $\phi$ as the indicator function of $\Delta(X, t, 1)$ and using the normalization of $\omega_{1}$ and $\omega_{2}$ we get that $\alpha_{1}=\alpha_{2}$. Therefore $u_{1} \equiv u_{2}$ and $\omega_{1} \equiv \omega_{2}$. 
To prove the existence we argue as in the proof of Lemma 13. We let $(X, t) \in \partial \Omega$ and define $R>0$ to be a large positive number. Let $\left(\hat{X}_{j}, \hat{t}_{j}\right) \in$ $\Gamma_{A}^{+}\left(X, t, 2^{j} R\right)$ for $j=1,2, \ldots$ and define for $(Z, \tau) \in C_{R}(X, t) \cap \Omega$

$$
u_{j}(Z, \tau)=\frac{G\left(\hat{X}_{j}, \hat{t}_{j}, Z, \tau\right)}{G\left(\hat{X}_{j}, \hat{t}_{j}, A_{1}(X, t)\right)} .
$$

Let $\phi \in C_{0}^{\infty}\left(C_{R}(X, t)\right)$ and let as usual $\omega\left(\hat{X}_{j}, \hat{t}_{j}, \cdot\right)$ be the caloric measure defined with respect to $\left(\hat{X}_{j}, \hat{t}_{j}\right)$. Then

$$
\begin{array}{rl}
\int_{\partial \Omega} \phi(Z, \tau) G\left(\hat{X}_{j}, \hat{t}_{j}, A_{1}(X, t)\right)^{-1} & d \omega\left(\hat{X}_{j}, \hat{t}_{j}, Z, \tau\right) \\
& =\int_{\Omega} u_{j}(Z, \tau)\left(\Delta-\partial_{\tau}\right) \phi(Z, \tau) d Z d \tau .
\end{array}
$$

Defining measures

$$
d \mu_{j}(Z, \tau)=G\left(\hat{X}_{j}, \hat{t}_{j}, A_{1}(X, t)\right)^{-1} d \omega\left(\hat{X}_{j}, \hat{t}_{j}, Z, \tau\right)
$$

we can conclude that

$$
\int_{\partial \Omega} \phi(Z, \tau) d \mu_{j}(Z, \tau)=\int_{\Omega} u_{j}(Z, \tau)\left(\Delta-\partial_{\tau}\right) \phi(Z, \tau) d Z d \tau
$$

for all $\phi \in C_{0}^{\infty}\left(C_{R}(X, t)\right)$. Using Lemma 7 and the fact that $G\left(\hat{X}_{j}, \hat{t}_{j}, \cdot\right)$ satisfies a strong Harnack inequality in $C_{R}(X, t) \cap \Omega$ we have that

$$
\begin{aligned}
\mu_{j}(\Delta(X, t, R))=\frac{\omega\left(\hat{X}_{j}, \hat{t}_{j}, \Delta(X, t, R)\right)}{G\left(\hat{X}_{j}, \hat{t}_{j}, A_{1}(X, t)\right)} & \sim \frac{R^{n} G\left(\hat{X}_{j}, \hat{t}_{j}, A_{R}(X, t)\right)}{G\left(\hat{X}_{j}, \hat{t}_{j}, A_{1}(X, t)\right)} \\
& \sim R^{n} u_{j}(Z, \tau)
\end{aligned}
$$

for all $(Z, \tau) \in C_{R}(X, t) \cap \Omega .\left\{u_{j}\right\}$ is, as in the proof of Lemma 13, a uniformly bounded set of functions on $\Omega \cap C_{R}(X, t)$. Hence the sequence $\left\{\mu_{j}\right\}$ is a uniformly bounded set of measures on $C_{R}(X, t) \cap \partial \Omega$ and therefore there exists a subsequence $\left\{\mu_{\tilde{j}_{k}}\right\}$ and a Radon measure $\mu$ such that,

$$
\int_{\partial \Omega} \phi(Z, \tau) d \mu_{\tilde{j}_{k}}(Z, \tau) \rightarrow \int_{\partial \Omega} \phi(Z, \tau) d \mu(Z, \tau),
$$

for all $\phi \in C_{0}^{\infty}\left(C_{R}(X, t)\right)$ as $k \rightarrow \infty$. If we again choose a sequence of numbers $R_{i}$ such that $R_{i} \rightarrow \infty$ and pick a diagonal subsequence we can therefore 
conclude that there exists a subsequence $\left\{j_{k}\right\}$ such that $\left\{\mu_{j_{k}}\right\}$ converges to a Radon measure $\mu$ such that for all $\phi \in C_{0}^{\infty}\left(\mathrm{R}^{n+1}\right)$,

$$
\int_{\partial \Omega} \phi(Z, \tau) d \mu_{j_{k}}(Z, \tau) \rightarrow \int_{\partial \Omega} \phi(Z, \tau) d \mu(Z, \tau),
$$

as $k \rightarrow \infty$. Repeating the argument of Lemma 13 we can also conclude that $\left\{u_{j_{k}}\right\}$ converges, uniformly on compacts subsets, to a non-negative solution $u_{\infty}$ and for all $\phi \in C_{0}^{\infty}\left(\mathrm{R}^{n+1}\right)$

$$
\int_{\partial \Omega} \phi(Z, \tau) d \mu(Z, \tau)=\int_{\Omega} u_{\infty}(Z, \tau)\left(\Delta-\partial_{\tau}\right) \phi d Z d \tau .
$$

Define $\omega_{\infty}=\mu / \mu(\Delta(X, t, 1))$ and $u_{\infty}=u_{\infty} / \mu(\Delta(X, t, 1))$. Then for all $\phi \in C_{0}^{\infty}\left(\mathrm{R}^{n+1}\right)$

$$
\int_{\partial \Omega} \phi(Z, \tau) d \omega_{\infty}(Z, \tau)=\int_{\Omega} u_{\infty}(Z, \tau)\left(\Delta-\partial_{\tau}\right) \phi d Z d \tau .
$$

This completes the existence part of the proof. Left is to prove that $\omega_{\infty}$ is a doubling measure. But if $(\tilde{X}, \tilde{t}) \in \partial \Omega$ and $r>0$, then it follows from Lemma 9 that

$$
\begin{aligned}
\omega_{\infty}(\Delta(\tilde{X}, \tilde{t}, 2 r)) & \leq \liminf _{j_{k} \rightarrow \infty} \frac{\omega\left(\hat{X}_{j_{k}}, \hat{t}_{j_{k}}, \Delta(\tilde{X}, \tilde{t}, 2 r)\right)}{\mu(\Delta(X, t, 1)) G\left(\hat{X}_{j_{k}}, \hat{t}_{j_{k}}, A_{1}(X, t)\right)} \\
& \leq C \liminf _{j_{k} \rightarrow \infty} \frac{\omega\left(\hat{X}_{j_{k}}, \hat{t}_{j_{k}}, \Delta(\tilde{X}, \tilde{t}, r / 2)\right)}{\mu(\Delta(X, t, 1)) G\left(\hat{X}_{j_{k}}, \hat{t}_{j_{k}}, A_{1}(X, t)\right)} \\
& \leq C \omega_{\infty}(\Delta(\tilde{X}, \tilde{t}, r)) .
\end{aligned}
$$

This completes the proof.

\section{A blow-up argument and the classification of global solutions}

In the following we let $\Omega$ be a two-sided NTA-domain with Ahlfors regular boundary. Let $\left(X_{j}, t_{j}\right) \in \partial \Omega \rightarrow(\tilde{X}, \tilde{t}) \in \partial \Omega$ and assume that $(\tilde{X}, \tilde{t})=(0,0)$. For a sequence $\left\{r_{j}\right\}$ of real numbers tending to zero we define,

$$
\Omega_{j}=\left\{\left(r_{j}^{-1}\left(X-X_{j}\right), r_{j}^{-2}\left(t-t_{j}\right)\right):(X, t) \in \Omega\right\} .
$$

This section is devoted to the analysis of these blow-ups by making use of our assumption on the caloric Poisson kernels $k^{i}\left(\hat{X}^{i}, \hat{t}^{i}, \cdot\right)$ and we will therefore also assume that $t_{j}<\hat{t}^{2}<\hat{t}^{1}$ for all $j$. 


\subsection{Blow-ups}

Recall that the parabolic distance between the two sets $F_{1}, F_{2}$ is defined as

$$
d\left(F_{1}, F_{2}\right)=\inf \left\{|X-Y|+|s-t|^{1 / 2}:(X, t) \in F_{1},(Y, s) \in F_{2}\right\} .
$$

Based on this we introduce the parabolic Hausdorff distance between two sets $F_{1}, F_{2}$ as

$$
D\left(F_{1}, F_{2}\right)=\sup \left\{d\left(x, F_{2}\right): x \in F_{1}\right\}+\sup \left\{d\left(F_{1}, y\right): y \in F_{2}\right\} .
$$

In the following we will consider uniform Hausdorff convergence (in the metric induced by the parabolic Hausdorff distance) on compact sets. To define this properly we consider a sequence of closed sets $\left\{F_{j}\right\}_{j}, F_{j} \subset \mathrm{R}^{n+1}$. We say that $F_{j}$ converges to a closed set $F \subset \mathrm{R}^{n+1}$ in the parabolic Hausdorff distance sense, uniformly on compact subsets of $\mathrm{R}^{n+1}$, if for any compact set $K \subset \mathrm{R}^{n+1}$ and any $\epsilon>0$ there exists $j_{0} \geq 1$ so that if $j \geq j_{0}$ then

$$
D\left(F_{j} \cap K, F \cap K\right)<\epsilon .
$$

Furthermore a sequence of open sets $\left\{E_{j}\right\}_{j}, E_{j} \subset \mathrm{R}^{n+1}$, is said to converge to an open set $E \subset \mathrm{R}^{n+1}$ in the parabolic Hausdorff distance sense uniformly on compact subsets of $\mathrm{R}^{n+1}$ if $\mathrm{R}^{n+1} \backslash E_{j} \rightarrow \mathrm{R}^{n+1} \backslash E$ in the parabolic Hausdorff distance sense uniformly on compact subsets of $\mathrm{R}^{n+1}$.

In the following blow-up argument we will explore the information contained in the condition $\log k(\hat{X}, \hat{t}, \cdot) \in \operatorname{VMO}(d \sigma)$. To do so we define a kernel $k_{j}$ on $\partial \Omega_{j}$, related to the Poisson kernel $k$ on $\partial \Omega$, as

$$
k_{j}(Y, s)=\frac{\sigma\left(\Delta\left(X_{j}, t_{j}, r_{j}\right)\right) k\left(\hat{X}, \hat{t}, X_{j}+r_{j} Y, t_{j}+r_{j}^{2} s\right)}{\omega\left(\hat{X}, \hat{t}, \Delta\left(X_{j}, t_{j}, r_{j}\right)\right)} .
$$

We will refer to $k_{j}$ as the Poisson kernel on $\partial \Omega_{j}$. We also define

$$
u_{j}(Z, \tau)=\frac{\sigma\left(\Delta\left(X_{j}, t_{j}, r_{j}\right)\right) G\left(\hat{X}, \hat{t}, X_{j}+r_{j} Z, t_{j}+r_{j}^{2} \tau\right)}{r_{j} \omega\left(\hat{X}, \hat{t}, \Delta\left(X_{j}, t_{j}, r_{j}\right)\right)}
$$

whenever $(Z, \tau) \in \Omega_{j}$. Then $u_{j}$ is adjoint caloric in $\Omega_{j}$ outside of its pole and it is zero on $\partial \Omega_{j} . u_{j}$ can be refered to as the Green function associated to the kernel $k_{j}$. Finally we define the associated caloric measure through $d w_{j}=k_{j}(Y, s) d \sigma_{j}(Y, s)$ and we note that for arbitrary Borel sets $E \subset \mathbf{R}^{n+1}$

$$
\omega_{j}(E)=\int_{E} k_{j}(Y, s) d \sigma_{j}(Y, s)=r_{j}^{-n-1} \sigma\left(\Delta\left(X_{j}, t_{j}, r_{j}\right)\right) \tilde{\omega}_{j}(E)
$$


where

$$
\tilde{\omega}_{j}(E)=\frac{\omega\left(\hat{X}, \hat{t},\left\{(Z, \tau) \in \partial \Omega:\left(\left(Z-X_{j}\right) / r_{j},\left(\tau-t_{j}\right) / r_{j}^{2}\right) \in E\right\}\right)}{\omega\left(\hat{X}, \hat{t}, \Delta\left(X_{j}, t_{j}, r_{j}\right)\right)} .
$$

We will start by proving the following two lemmas.

Lemma 15 . Let $\Omega$ be a two-sided NTA-domain with Ahlfors regular boundary. Let $\left(X_{j}, t_{j}\right) \in \partial \Omega \rightarrow(\tilde{X}, \tilde{t}) \in \partial \Omega$ and assume that $(\tilde{X}, \tilde{t})=(0,0)$. For a sequence $\left\{r_{j}\right\}$ of real numbers tending to zero we define,

$$
\Omega_{j}=\left\{\left(r_{j}^{-1}\left(X-X_{j}\right), r_{j}^{-2}\left(t-t_{j}\right)\right):(X, t) \in \Omega\right\} .
$$

Then $\Omega_{j} \rightarrow \Omega_{\infty}$ and $\partial \Omega_{j} \rightarrow \partial \Omega_{\infty}$ in the parabolic Hausdorff distance sense, uniformly on compact subsets of $\mathrm{R}^{n+1}$, as $j \rightarrow \infty$. Furthermore, $\Omega_{\infty}$ is a two-sided NTA-domain with Ahlfors regular boundary.

Lemma 16. Let $\Omega_{j}$ and $\Omega_{\infty}$ be as in Lemma 15. Then $u_{j} \rightarrow u_{\infty}$ uniformly on compact subsets, $u_{\infty}$ is a positive adjoint caloric function in $\Omega_{\infty}$ and $u_{\infty}=0$ on $\partial \Omega_{\infty}$. Moreover $\omega_{j} \rightarrow \omega_{\infty}$ weakly as Radon measures and for all $\phi \in C_{0}^{\infty}\left(\mathrm{R}^{n+1}\right)$

$$
\int_{\partial \Omega} \phi(Y, s) d \omega_{\infty}(Y, s)=\int_{\Omega} u_{\infty}(Y, s)\left(\Delta-\partial_{s}\right) \phi(Y, s) d Y d s .
$$

In particular $\omega_{\infty}$ is the caloric measure of $\Omega_{\infty}$ at infinity.

We start by proving Lemma 15.

Proof of Lemma 15. Note that for each $j \geq 1,(0,0) \in \partial \Omega_{j}$ and $C_{1}(0,0) \cap$ $\Omega_{j} \neq \emptyset$. Using this we can conclude that given a compact set $K \subset \mathrm{R}^{n+1}$ there exists a subsequence $\left\{\tilde{j}_{m}\right\}_{m}$ such that $K \cap \partial \Omega_{\tilde{j}_{m}}$ and $K \cap \Omega_{\tilde{j}_{m}}$ converge in the parabolic Hausdorff distance sense. We can therefore exhaust $\mathrm{R}^{n+1}$ by a sequence of compact sets in order to ensure that there exists a subsequence $\left\{j_{m}\right\}_{m}$ such that $\partial \Omega_{j_{m}}$ and $\Omega_{j_{m}}$ converge in the parabolic Hausdorff distance sense uniformly on compact sets. Hence by an appropriate relabeling we can conclude that as $j \rightarrow \infty, \Omega_{j} \rightarrow \Omega_{\infty}, \partial \Omega_{j} \rightarrow \Sigma_{\infty}$ in the parabolic Hausdorff distance sense uniformly on compact subsets of $\mathrm{R}^{n+1}$. In analogy with the proof of Theorem 4.1 in [14] we want to prove that $\partial \Omega_{\infty}=\Sigma_{\infty}$ and that $\Omega_{\infty}$ is a two-sided NTA-domain with Ahlfors regular boundary.

Note that since $\omega$ is a doubling measure we have that for any compact set $K \subset \mathrm{R}^{n+1}, \sup _{j \geq 1} \tilde{\omega}_{j}(K) \leq C_{K}$. Hence arguing as in the proof of Lemma 14 there exists a subsequence (which we relabel) such that $\tilde{\omega}_{j} \rightarrow \tilde{\omega}_{\infty}$ in the sense that

$$
\int \phi d \tilde{\omega}_{j} \rightarrow \int \phi d \tilde{\omega}_{\infty}
$$


for all $\phi \in C_{0}^{\infty}\left(\mathrm{R}^{n+1}\right)$. We will start by proving that the support of $\tilde{\omega}_{\infty}$ equals $\Sigma_{\infty}$. To do this we let $(\hat{Z}, \hat{\tau}) \in \Sigma_{\infty}$. Again by construction there exists a sequence $\left(Z_{j}, \tau_{j}\right)$ such that $\left(Z_{j}, \tau_{j}\right) \in \partial \Omega$ and

$$
\left(\tilde{Z}_{j}, \tilde{\tau}_{j}\right):=\left(\left(Z_{j}-X_{j}\right) / r_{j},\left(\tau_{j}-t_{j}\right) / r_{j}^{2}\right) \rightarrow(\hat{Z}, \hat{\tau}) .
$$

Furthermore for every $r \in(0,1)$ there exists $j_{0} \geq 1$ such that for $j \geq j_{0}$, $d\left(\left(\tilde{Z}_{j}, \tilde{\tau}_{j}\right),(\hat{Z}, \hat{\tau})\right)<r / 2$ and $d\left(\left(Z_{j}, \tau_{j}\right),\left(X_{j}, t_{j}\right)\right)<C r_{j}$ for some large $C=$ $C(\hat{Z}, \hat{\tau})$. As $\omega$ is a doubling measure,

$$
\begin{aligned}
\tilde{\omega}_{j}\left(C_{r}(\hat{Z}, \hat{\tau})\right) \\
\quad=\frac{\omega\left(\hat{X}, \hat{t},\left\{(Z, \tau) \in \partial \Omega:\left(\left(Z-X_{j}\right) / r_{j},\left(\tau-t_{j}\right) / r_{j}^{2}\right) \in C_{r}(\hat{Z}, \hat{\tau})\right\}\right)}{\omega\left(\hat{X}, \hat{t}, \Delta\left(X_{j}, t_{j}, r_{j}\right)\right)} \\
\quad \geq \frac{\omega\left(\hat{X}, \hat{t}, C_{r r_{j} / 2}\left(Z_{j}, \tau_{j}\right)\right)}{\omega\left(\hat{X}, \hat{t}, \Delta\left(X_{j}, t_{j}, r_{j}\right)\right)} \geq \frac{\omega\left(\hat{X}, \hat{t}, C_{r_{j} / 2}\left(Z_{j}, \tau_{j}\right)\right)}{\omega\left(\hat{X}, \hat{t}, C_{2 C r_{j}}\left(Z_{j}, \tau_{j}\right)\right)} \geq \tilde{C}(r, C) .
\end{aligned}
$$

This implies that if $(\hat{Z}, \hat{\tau}) \in \Sigma_{\infty}$ then $(\hat{Z}, \hat{\tau})$ is in the support of $\tilde{\omega}_{\infty}$. Left is to prove the other inclusion. I.e., in this case we start by assuming that $(\hat{Z}, \hat{\tau})$ is in the support of $\tilde{\omega}_{\infty}$. We want to prove that there exists $\left(Z_{j}, \tau_{j}\right) \in \partial \Omega$ such that

$$
\left(\tilde{Z}_{j}, \tilde{\tau}_{j}\right):=\left(\left(Z_{j}-X_{j}\right) / r_{j},\left(\tau_{j}-t_{j}\right) / r_{j}^{2}\right) \rightarrow(\hat{Z}, \hat{\tau}) .
$$

If this is not the case then there exists $\epsilon>0$ and $j_{0}$ such that for any sequence $\left(Z_{j}, \tau_{j}\right) \in \partial \Omega$ as above $d\left(\left(\tilde{Z}_{j}, \tilde{\tau}_{j}\right),(\hat{Z}, \hat{\tau})\right) \geq \epsilon$ if $j \geq j_{0}$. In particular in this case $C_{\epsilon / 2}(\hat{Z}, \hat{\tau}) \cap \partial \Omega_{j}=\emptyset$. If we take $\phi \in C_{0}^{\infty}\left(C_{\epsilon / 2}(\hat{Z}, \hat{\tau})\right)$ we then get as $\tilde{\omega}_{j} \rightarrow \tilde{\omega}_{\infty}$ that

$$
0=\int \phi(Y, s) d \tilde{\omega}_{j}(Y, s) \rightarrow \int \phi(Y, s) d \tilde{\omega}_{\infty}(Y, s)
$$

I.e.,

$$
\int \phi(Y, s) d \tilde{\omega}_{\infty}(Y, s)=0
$$

for all $\phi \in C_{0}^{\infty}\left(C_{\epsilon / 2}(\hat{Z}, \hat{\tau})\right)$. This contradicts the assumption that $(\hat{Z}, \hat{\tau})$ is in the support of $\tilde{\omega}_{\infty}$. I.e., $(\hat{Z}, \hat{\tau}) \in \Sigma_{\infty}$ and we can conclude that the support of $\tilde{\omega}$ coincides with $\Sigma_{\infty}$. Also note that trivially $\omega_{j} \rightarrow \omega_{\infty}$ and the support of $\omega_{\infty}$ coincides with $\Sigma_{\infty}$.

We now prove that $\partial \Omega_{\infty} \subset \Sigma_{\infty}$. To do this we let $(Z, \tau) \in \partial \Omega_{\infty}=$ $\overline{\Omega_{\infty}} \cap \overline{\mathrm{R}^{n+1} \backslash \Omega_{\infty}}$ and note that given $\epsilon>0$, there exist $(Y, s) \in \Omega_{\infty} \cap C_{\epsilon}(Z, \tau)$ 
and $(\hat{Y}, \hat{s}) \in\left[\mathrm{R}^{n+1} \backslash \overline{\Omega_{\infty}}\right] \cap C_{\epsilon}(Z, \tau)$. There also exist sequencies of points $\left(Y_{j}, s_{j}\right) \in \Omega$ and $\left(\hat{Y}_{j}, \hat{s}_{j}\right) \in\left[\mathrm{R}^{n+1} \backslash \bar{\Omega}\right]$ such that

$$
\begin{aligned}
& (Y, s)=\left(\left(Y_{j}-X_{j}\right) / r_{j},\left(s_{j}-t_{j}\right) / r_{j}^{2}\right), \\
& (\hat{Y}, \hat{s})=\left(\left(\hat{Y}_{j}-X_{j}\right) / r_{j},\left(\hat{s}_{j}-t_{j}\right) / r_{j}^{2}\right) .
\end{aligned}
$$

Let $l_{j}$ be the parabolic line connecting $\left(Y_{j}, s_{j}\right)$ and $\left(\hat{Y}_{j}, \hat{s}_{j}\right)$ and pick $\left(Z_{j}, \tau_{j}\right) \in$ $l_{j} \cap \partial \Omega$. As $\partial \Omega$ separates $\mathrm{R}^{n+1}$ at least one such point exists. As $\left\{\left(Z_{j}-X_{j}\right) / r_{j}\right\}_{j}$ as well as $\left\{\left(\tau_{j}-t_{j}\right) / r_{j}^{2}\right\}_{j}$ are bounded sequences there exist a subsequence (which we relabel) such that

$$
\left(\left(Z_{j}-X_{j}\right) / r_{j},\left(\tau_{j}-t_{j}\right) / r_{j}^{2}\right) \rightarrow(\hat{Z}, \hat{\tau}) \in \Sigma_{\infty} .
$$

Furthermore as

$$
\begin{aligned}
d\left(\left(\left(Z_{j}-X_{j}\right) / r_{j},\left(\tau_{j}-t_{j}\right) / r_{j}^{2}\right),\left(\left(Y_{j}-X_{j}\right) / r_{j},\left(s_{j}\right.\right.\right. & \left.\left.\left.-t_{j}\right) / r_{j}^{2}\right)\right) \\
& \leq r_{j}^{-1} d\left(\left(Y_{j}, s_{j}\right),\left(\hat{Y}_{j}, \hat{s}_{j}\right)\right)
\end{aligned}
$$

we can conclude, by letting $j \rightarrow \infty$, that

$$
d((Y, s),(\hat{Z}, \hat{\tau})) \leq d((Y, s),(\hat{Y}, \hat{s})) \leq C \epsilon
$$

for a universial constant $C$. By the same line of thought

$$
\begin{aligned}
d((Z, \tau),(\hat{Z}, \hat{\tau})) & \leq d((Z, \tau),(\hat{Y}, \hat{s}))+d((\hat{Z}, \hat{\tau}),(\hat{Y}, \hat{s})) \\
& \leq d((Z, \tau),(\hat{Y}, \hat{s}))+d((Y, s),(\hat{Y}, \hat{s})) \\
& \leq \tilde{C} \epsilon .
\end{aligned}
$$

In total we have proved that for any $(Z, \tau) \in \partial \Omega_{\infty}$ and for any $\epsilon>0$ there exists $(\hat{Z}, \hat{\tau}) \in \Sigma_{\infty}$ such that $d((Z, \tau),(\hat{Z}, \hat{\tau})) \leq \epsilon$. This argument proves that $(Z, \tau)$ is in the closure of the set $\Sigma_{\infty}$. But the closure of the set $\Sigma_{\infty}$ equals, as we have proven above, the closure of the support of $\omega_{\infty}$. The latter equals the support of $\omega_{\infty}$ as the support is closed. Based on this we can conclude that $(Z, \tau) \in \Sigma_{\infty}$ and that $\partial \Omega_{\infty} \subset \Sigma_{\infty}$.

Left is to prove that $\Sigma_{\infty} \subset \partial \Omega_{\infty}$. We let $(\hat{Z}, \hat{\tau}) \in \Sigma_{\infty}$. By construction there exists a sequence $\left(Z_{j}, \tau_{j}\right)$ such that $\left(Z_{j}, \tau_{j}\right) \in \partial \Omega$ and

$$
\left(\tilde{Z}_{j}, \tilde{\tau}_{j}\right):=\left(\left(Z_{j}-X_{j}\right) / r_{j},\left(\tau_{j}-t_{j}\right) / r_{j}^{2}\right) \rightarrow(\hat{Z}, \hat{\tau}) .
$$

In order to argue as in the proof of Theorem 4.1 in [14] we start by fixing $M$ and by considering arbitrary $\rho>0$. As $\Omega$ is a two-sided NTA-domain we can 
assume that there exist points $A_{\rho r_{j}}\left(Z_{j}, \tau_{j}\right) \in \Omega$ and $\tilde{A}_{\rho r_{j}}\left(Z_{j}, \tau_{j}\right) \in \mathrm{R}^{n+1} \backslash \bar{\Omega}$ such that if $M$ is large enough then

$$
\begin{aligned}
& C_{\rho r_{j} / M}\left(A_{\rho r_{j}}\left(Z_{j}, \tau_{j}\right)\right) \subset \Omega, \quad d\left(A_{\rho r_{j}}\left(Z_{j}, \tau_{j}\right),\left(Z_{j}, \tau_{j}\right)\right) \leq \rho r_{j}, \\
& C_{\rho r_{j} / M}\left(\tilde{A}_{\rho r_{j}}\left(Z_{j}, \tau_{j}\right)\right) \subset \mathrm{R}^{n+1} \backslash \bar{\Omega}, \quad d\left(\tilde{A}_{\rho r_{j}}\left(Z_{j}, \tau_{j}\right),\left(Z_{j}, \tau_{j}\right)\right) \leq \rho r_{j} .
\end{aligned}
$$

By applying the blow up argument we can therefore construct $A_{j}(\rho) \in \Omega_{j}$ and $\tilde{A}_{j}(\rho) \in \mathrm{R}^{n+1} \backslash \bar{\Omega}_{j}$ such that

$$
\begin{aligned}
& C_{\rho / M}\left(A_{j}(\rho)\right) \subset \Omega_{j}, \quad d\left(A_{j}(\rho),\left(\tilde{Z}_{j}, \tilde{\tau}_{j}\right)\right) \leq \rho, \\
& C_{\rho / M}\left(\tilde{A}_{j}(\rho)\right) \subset \mathrm{R}^{n+1} \backslash \bar{\Omega}_{j}, \quad d\left(\tilde{A}_{j}(\rho),\left(\tilde{Z}_{j}, \tilde{\tau}_{j}\right)\right) \leq \rho .
\end{aligned}
$$

Going to the limit we can conclude that, for every $\rho>0$, there exist points $A_{\infty}(\rho) \in \Omega_{\infty}$ and $\tilde{A}_{\infty}(\rho) \in \mathrm{R}^{n+1} \backslash \bar{\Omega}_{\infty}$ such that

$$
\begin{aligned}
& C_{\rho / M}\left(A_{\infty}(\rho)\right) \subset \Omega_{\infty}, \quad d\left(A_{\infty}(\rho),(\hat{Z}, \hat{\tau})\right) \leq \rho, \\
& C_{\rho / M}\left(\tilde{A}_{\infty}(\rho)\right) \subset \mathrm{R}^{n+1} \backslash \bar{\Omega}_{\infty}, \quad d\left(\tilde{A}_{\infty}(\rho),(\hat{Z}, \hat{\tau})\right) \leq \rho .
\end{aligned}
$$

If we let $\rho \rightarrow 0$ we can conclude that $(\hat{Z}, \hat{\tau}) \in \partial \Omega_{\infty}$ and hence that $\Sigma_{\infty} \subset$ $\partial \Omega_{\infty}$. In total we have proven that $\Sigma_{\infty}=\partial \Omega_{\infty}$.

By essentially repeating the argument above for the proof that $\Sigma_{\infty} \subset \partial \Omega_{\infty}$ we realize that $\Omega_{\infty}$ defines a two-sided NTA-domain. What remains is to prove that $\partial \Omega_{\infty}$ is Ahlfors regular. Let $j \geq 1$ and let $F \subset \mathrm{R}^{n+1}$ be a Borel set. Also let $\bar{F}, \partial F$ denote the closure and the boundary of $F$ respectively. We define $\sigma_{j}(F)=\int_{F \cap \partial \Omega_{j}} d \sigma_{j}(t) d t$ where $d \sigma_{j}(t)$ is the $n-1$ dimensional Hausdorff measure on the time slice $F \cap \partial \Omega_{j} \cap\left(\mathrm{R}^{n} \times\{t\}\right)$. Similarly we define $\sigma_{\infty}(F)=\int_{F \cap \partial \Omega_{\infty}} d \sigma_{\infty}(t) d t$ where $d \sigma_{\infty}(t)$ is the $n-1$ dimensional Hausdorff measure on the time slice $F \cap \partial \Omega_{\infty} \cap\left(\mathrm{R}^{n} \times\{t\}\right)$. Let $(\hat{Z}, \hat{\tau}) \in \partial \Omega_{\infty}$ and let $r>0$. By construction there exists a sequence $\left(Z_{j}, \tau_{j}\right)$ such that $\left(Z_{j}, \tau_{j}\right) \in \partial \Omega$ and

$$
\left(\tilde{Z}_{j}, \tilde{\tau}_{j}\right):=\left(\left(Z_{j}-X_{j}\right) / r_{j},\left(\tau_{j}-t_{j}\right) / r_{j}^{2}\right) \rightarrow(\hat{Z}, \hat{\tau}) .
$$

Note that by the Ahlfors regularity of $\partial \Omega_{j}$ we get

$$
\begin{aligned}
\sigma_{j}\left(C_{r}(\hat{Z}, \hat{\tau})\right) & \leq \sigma_{j}\left(C_{r+d\left(\left(\tilde{Z}_{j}, \tilde{\tau}_{j}\right),(\hat{Z}, \hat{\tau})\right)}\left(\tilde{Z}_{j}, \tilde{\tau}_{j}\right)\right) \\
& \leq C\left(r+d\left(\left(\left(\tilde{Z}_{j}, \tilde{\tau}_{j}\right),(\hat{Z}, \hat{\tau})\right)\right)^{n+1} .\right.
\end{aligned}
$$

Hence

$$
\liminf _{j \rightarrow \infty} \sigma_{j}\left(C_{r}(\hat{Z}, \hat{\tau})\right) \leq C r^{n+1}
$$


Therefore as

$$
\sigma_{\infty}\left(C_{r}(\hat{Z}, \hat{\tau})\right) \leq \lim \inf _{j \rightarrow \infty} \sigma_{j}\left(C_{r}(\hat{Z}, \hat{\tau})\right) \leq C r^{n+1}
$$

we have the proof in one direction.

To focus on the proof in the other direction for $(\hat{Z}, \hat{\tau}) \in \partial \Omega_{\infty}$ we let $A=A_{r}(\hat{Z}, \hat{\tau})=\left(A_{r}^{x}(\hat{Z}, \hat{\tau}), \hat{\tau}\right), \tilde{A}=\tilde{A}_{r}(\hat{Z}, \hat{\tau})=\left(\tilde{A}_{r}^{x}(\hat{Z}, \hat{\tau}), \hat{\tau}\right)$ be points such that $A \in \Omega_{\infty}, \tilde{A} \in \mathrm{R}^{n+1} \backslash \bar{\Omega}_{\infty}$ and $d\left(A, \partial \Omega_{\infty}\right) \sim r \sim d(A,(\hat{Z}, \hat{\tau}))$, $d\left(\tilde{A}, \partial \Omega_{\infty}\right) \sim r \sim d(\tilde{A},(\hat{Z}, \hat{\tau}))$. Define $A^{*}=\left(\left(\tilde{A}_{r}^{x}(\hat{Z}, \hat{\tau})+\tilde{A}_{r}^{x}(\hat{Z}, \hat{\tau})\right) / 2, \hat{\tau}\right)$. Let $M$ be a large positive number. Let $P$ be the plane, which contains a line parallel to the time-axis and the point $A^{*}$ and which is perpendicular to the line $A-\tilde{A}$. By $\pi(Z, \tau)$ we denote the orthogonal projection of $(Z, \tau)$ to the plane $P$. A simple geometric argument gives that $\pi\left(C_{r}(\hat{Z}, \hat{\tau}) \cap \Omega\right)$ contains at least the projection of a parabolic cylinder $C_{r / \hat{M}}(\hat{X}, \hat{t})$ for some universial $M$ and for some point $(\hat{X}, \hat{t})$. An easy consequence of this is that

$$
\sigma_{\infty}\left(C_{r}(\hat{Z}, \hat{\tau})\right) \geq C^{-1} r^{n+1} .
$$

This completes the proof of the lemma.

To continue we proceed with the proof of Lemma 16.

ProOf OF Lemma 16. Recall that from the argument of proof of Lemma 15 it follows that $\omega_{j} \rightarrow \omega_{\infty}$ and that the support of $\omega_{\infty}$ coincides with $\partial \Omega_{\infty}$. Let $\phi \in C_{0}^{\infty}\left(\mathrm{R}^{n+1} \backslash(\hat{X}, \hat{t})\right)$ and define $\phi_{j}(Y, s)=\phi\left(r_{j}^{-1}\left(Y-X_{j}\right), r_{j}^{-2}\left(s-t_{j}\right)\right)$. By the Riesz representation formula we have

$$
\int_{\partial \Omega} \phi_{j}(Y, s) d \omega(\hat{X}, \hat{t}, Y, s)=\int_{\Omega} G(\hat{X}, \hat{t}, Z, \tau)\left(\Delta \phi_{j}-\partial_{\tau} \phi_{j}\right) d Z d \tau .
$$

If we let $\left(\hat{X}_{j}, \hat{t}_{j}\right)=\left(r_{j}^{-1}\left(\hat{X}-X_{j}\right), r_{j}^{-2}\left(\hat{t}-t_{j}\right)\right)$, then by a change of variables,

$$
\int_{\partial \Omega_{j}} \phi(Y, s) d \omega_{j}\left(\hat{X}_{j}, \hat{t}_{j}, Y, s\right)=\int_{\Omega_{j}} u_{j}(Z, \tau)\left(\Delta \phi-\partial_{\tau} \phi\right) d Z d \tau
$$

where $\omega_{j}$ and $u_{j}$ were introduced above the statement of Lemma 15. Defining $u_{j} \equiv 0$ on the complement of $\Omega_{j}$ we can conclude, using the same argument as in the proof of Lemma 13, that $\left\{u_{j}\right\}$ is a uniformly bounded sequence on compacts. By the Arzela-Ascoli theorem $u_{j} \rightarrow u_{\infty}$ uniformly on compact subsets and $u_{\infty}$ is a positive adjoint caloric function in $\Omega_{\infty}$ such that $u_{\infty}=0$ on $\partial \Omega_{\infty}$. By weak convergence we can therefore conclude that

$$
\int_{\partial \Omega_{\infty}} \phi(Y, s) d \omega_{\infty}(Y, s)=\int_{\Omega_{\infty}} u_{\infty}(Y, s)\left(\Delta-\partial_{s}\right) \phi(Y, s) d Y d s
$$


for all $\phi \in C_{0}^{\infty}\left(\mathrm{R}^{n+1}\right)$.

In the following we will assume that $\Omega \subset \mathrm{R}^{n+1}$ is a two-sided NTA-domain with Ahlfors regular boundary and we define $\Omega^{1}=\Omega \subset \mathrm{R}^{n+1}, \Omega^{2}=\mathrm{R}^{n+1} \backslash \bar{\Omega}$. Let $\left(\hat{X}^{i}, \hat{t}^{i}\right) \in \Omega^{i}$, for $i \in\{1,2\}, \hat{t}^{2}<\hat{t}^{1}$ and define $\omega^{i}\left(\hat{X}^{i}, \hat{t}^{i}, \cdot\right)$ to be the caloric measures defined w.r.t. $\Omega^{1}$ and $\Omega^{2}$ respectively. In the following we will assume that $\omega^{i}\left(\hat{X}^{i}, \hat{t}^{i}, \cdot\right)$, for $i \in\{1,2\}$, is absolutely continuous with respect to the surface measure $\sigma$. We define the Poisson kernels as $k^{i}\left(\hat{X}^{i}, \hat{t}^{i}, \cdot\right)=$ $d \omega^{i}\left(\hat{X}^{i}, \hat{t}^{i}, \cdot\right) / d \sigma$.

Lemma 17. Assume that $\log k^{i}\left(\hat{X}^{i}, \hat{t}^{i}, \cdot\right) \in \operatorname{VMO}(d \sigma)$ for $i=1$ and $i=2$ and let $\omega_{j}^{i} \rightarrow \omega_{\infty}^{i}$ in the sense of Lemma 16. Then $d \omega_{\infty}^{1}=d \omega_{\infty}^{2}$ a.e. on $\partial \Omega_{\infty}$ and there exists a universial constant $C$ such that if $(X, t) \in \partial \Omega_{\infty}$ and $r>0$, then

$$
\omega_{\infty}^{i}(\Delta(X, t, r)) \leq C r^{n+1}
$$

Proof. To prove the lemma we will prove that for all $\phi \in C_{0}^{\infty}\left(\mathrm{R}^{n+1}\right)$, $\phi \geq 0$, we have

$$
\begin{aligned}
\int_{\partial \Omega_{\infty}} \phi d \omega_{\infty}^{1} & =\lim _{j \rightarrow \infty} \int_{\partial \Omega_{j}} \phi d \omega_{j}^{1}=\lim _{j \rightarrow \infty} \int_{\partial \Omega_{j}} \phi d \sigma_{j} \\
& =\lim _{j \rightarrow \infty} \int_{\partial \Omega_{j}} \phi d \omega_{j}^{2}=\int_{\partial \Omega_{\infty}} \phi d \omega_{\infty}^{2} .
\end{aligned}
$$

The second statement of the lemma also follows from this equality and the argument used in the proof of Lemma 15 . To do this we will only consider $i=1$ and we will in the following often instead of $\omega(\hat{X}, \hat{t}, \cdot)=\omega^{i}\left(\hat{X}^{1}, \hat{t}^{1}, \cdot\right)$ simply write $\omega(\cdot)$.

In the following we will assume that Lemma 12 is valid with $\tilde{\epsilon}=1 / 4$, i.e., there exists a constant $C=C(n, \Omega, A)$ such that for all $(X, t) \in \partial \Omega, r<r_{0}$, $|X-\hat{X}|^{2} \leq A(\hat{t}-t), \hat{t}-t \geq 8 r^{2}$ and $E \subset \Delta(X, t, r)$,

$$
C^{-1}\left(\frac{\sigma(E)}{\sigma(\Delta(X, t, r))}\right)^{5 / 4} \leq \frac{\omega(\hat{X}, \hat{t}, E)}{\omega(\hat{X}, \hat{t}, \Delta(X, t, r))} \leq C\left(\frac{\sigma(E)}{\sigma(\Delta(X, t, r))}\right)^{3 / 4} .
$$

Let $\phi \in C_{0}^{\infty}\left(\mathrm{R}^{n+1}\right)$ and recall that in our blow-up argument we considered a sequence of points $\left(X_{j}, t_{j}\right) \in \partial \Omega \rightarrow(\tilde{X}, \tilde{t}) \in \partial \Omega$ and a sequence of scales $\left\{r_{j}\right\}$, $r_{j} \rightarrow 0$, and we assumed for simplicity that $(\tilde{X}, \tilde{t})=(0,0)$. In the following we will assume that supp $\phi \subset C_{M}(0,0)$ for some $M>1$ and that $\phi \geq 0$. Let $\epsilon>0$ be given. As $\log k(\hat{X}, \hat{t}, \cdot) \in \operatorname{VMO}(d \sigma)$ there exists, by the JohnNirenberg inequality, $j_{0}$ such that for $j \geq j_{0}$, there exists $G_{j} \subset \Delta\left(X_{j}, t_{j}, M r_{j}\right)$, 


$$
\begin{aligned}
& \sigma\left(\Delta\left(X_{j}, t_{j}, M r_{j}\right)\right) \leq(1+\epsilon) \sigma\left(G_{j}\right) \text { and such that for every }(Y, s) \in G_{j} \\
& (1-\epsilon) \frac{1}{\sigma\left(\Delta\left(X_{j}, t_{j}, M r_{j}\right)\right)} \int_{\Delta\left(X_{j}, t_{j}, M r_{j}\right)} k d \sigma \leq k(Y, s) \\
& \quad \leq(1+\epsilon) \frac{1}{\sigma\left(\Delta\left(X_{j}, t_{j}, M r_{j}\right)\right)} \int_{\Delta\left(X_{j}, t_{j}, M r_{j}\right)} k d \sigma .
\end{aligned}
$$

Here $k=k(\cdot, \cdot)=k(\hat{X}, \hat{t}, \cdot, \cdot)$. We can also assume that $\left(X_{j}, t_{j}\right) \in C_{1}(0,0)$ for $j \geq j_{0}$. Using the inequality we have,

$$
(1-\epsilon) \leq \frac{\omega\left(\Delta\left(X_{j}, t_{j}, r_{j}\right) \cap G_{j}\right) \sigma\left(\Delta\left(X_{j}, t_{j}, M r_{j}\right)\right)}{\omega\left(\Delta\left(X_{j}, t_{j}, M r_{j}\right)\right) \sigma\left(\Delta\left(X_{j}, t_{j}, r_{j}\right) \cap G_{j}\right)} \leq(1+\epsilon) .
$$

In the following $C_{M}$ will denote constants which depend on $M$ and other parameters but are independent of $j$. Using these inequalities and the consequence of the VMO condition stated above, the constant $A$ can be chosen uniformly and independent of $j$ as $r_{j} \rightarrow 0$ and as the sequence $\left(X_{j}, t_{j}\right)$ converges to a point located below $(\hat{X}, \hat{t})$, we have

$$
\begin{aligned}
& \frac{\omega\left(\Delta\left(X_{j}, t_{j}, r_{j}\right)\right)}{\sigma\left(\Delta\left(X_{j}, t_{j}, r_{j}\right)\right)} \\
& =\frac{\omega\left(\Delta\left(X_{j}, t_{j}, r_{j}\right) \cap G_{j}\right)}{\sigma\left(\Delta\left(X_{j}, t_{j}, r_{j}\right)\right)}+\frac{\omega\left(\Delta\left(X_{j}, t_{j}, r_{j}\right) \backslash G_{j}\right)}{\sigma\left(\Delta\left(X_{j}, t_{j}, r_{j}\right)\right)} \\
& \leq(1+\epsilon) \frac{\sigma\left(\Delta\left(X_{j}, t_{j}, r_{j}\right) \cap G_{j}\right)}{\sigma\left(\Delta\left(X_{j}, t_{j}, r_{j}\right)\right)} \frac{\omega\left(\Delta\left(X_{j}, t_{j}, M r_{j}\right)\right)}{\sigma\left(\Delta\left(X_{j}, t_{j}, M r_{j}\right)\right)}+\frac{\omega\left(\Delta\left(X_{j}, t_{j}, r_{j}\right) \backslash G_{j}\right)}{\sigma\left(\Delta\left(X_{j}, t_{j}, r_{j}\right)\right)} \\
& \leq(1+\epsilon) \frac{\omega\left(\Delta\left(X_{j}, t_{j}, M r_{j}\right)\right)}{\sigma\left(\Delta\left(X_{j}, t_{j}, M r_{j}\right)\right)}+\frac{\omega\left(\Delta\left(X_{j}, t_{j}, r_{j}\right)\right.}{\sigma\left(\Delta\left(X_{j}, t_{j}, r_{j}\right)\right)}\left(\frac{\sigma\left(\Delta\left(X_{j}, t_{j}, r_{j}\right) \backslash G_{j}\right)}{\sigma\left(\Delta\left(X_{j}, t_{j}, r_{j}\right)\right)}\right)^{3 / 4} \\
& \leq(1+\epsilon) \frac{\omega\left(\Delta\left(X_{j}, t_{j}, M r_{j}\right)\right)}{\sigma\left(\Delta\left(X_{j}, t_{j}, M r_{j}\right)\right)}+C \epsilon^{3 / 4} M^{\frac{3}{4}(n+1)} \frac{\omega\left(\Delta\left(X_{j}, t_{j}, r_{j}\right)\right)}{\sigma\left(\Delta\left(X_{j}, t_{j}, r_{j}\right)\right)} .
\end{aligned}
$$


By similar deductions

$$
\begin{aligned}
\frac{\omega\left(\Delta\left(X_{j}, t_{j}, r_{j}\right)\right)}{\sigma\left(\Delta\left(X_{j}, t_{j}, r_{j}\right)\right)} & \geq \frac{\omega\left(\Delta\left(X_{j}, t_{j}, r_{j}\right) \cap G_{j}\right)}{\sigma\left(\Delta\left(X_{j}, t_{j}, r_{j}\right)\right)} \\
& \geq(1-\epsilon) \frac{\sigma\left(\Delta\left(X_{j}, t_{j}, r_{j}\right) \cap G_{j}\right)}{\sigma\left(\Delta\left(X_{j}, t_{j}, r_{j}\right)\right)} \frac{\omega\left(\Delta\left(X_{j}, t_{j}, M r_{j}\right)\right)}{\sigma\left(\Delta\left(X_{j}, t_{j}, M r_{j}\right)\right)} \\
& =(1-\epsilon)\left(1-\frac{\sigma\left(\Delta\left(X_{j}, t_{j}, r_{j}\right) \backslash G_{j}\right)}{\sigma\left(\Delta\left(X_{j}, t_{j}, r_{j}\right)\right)}\right) \frac{\omega\left(\Delta\left(X_{j}, t_{j}, M r_{j}\right)\right)}{\sigma\left(\Delta\left(X_{j}, t_{j}, M r_{j}\right)\right)} \\
& \geq(1-\epsilon)\left(1-C M^{n+1} \tilde{\epsilon}\right) \frac{\omega\left(\Delta\left(X_{j}, t_{j}, M r_{j}\right)\right)}{\sigma\left(\Delta\left(X_{j}, t_{j}, M r_{j}\right)\right)}
\end{aligned}
$$

To continue we define $\hat{G}_{j}=\left\{\left(r_{j}^{-1}\left(Y-X_{j}\right), r_{j}^{-2}\left(s-t_{j}\right)\right) ;(Y, s) \in G_{j}\right\}, \hat{F}_{j}=$ $\Delta(0,0, M) \backslash \hat{G}_{j}$. Using this notation we can conclude that for $(Y, s) \in \hat{G}_{j}$,

$$
(1-\epsilon) I_{j} \leq k_{j}(Y, s) \leq(1+\epsilon) I_{j}
$$

where

$$
I_{j}:=\frac{\sigma\left(\Delta\left(X_{j}, t_{j}, r_{j}\right)\right)}{\sigma\left(\Delta\left(X_{j}, t_{j}, M r_{j}\right)\right)} \frac{\omega\left(\Delta\left(X_{j}, t_{j}, M r_{j}\right)\right)}{\omega\left(\Delta\left(X_{j}, t_{j}, r_{j}\right)\right)}
$$

Based on the deductions above we get

$$
\begin{aligned}
I_{j}^{-1} & \leq \frac{\sigma\left(\Delta\left(X_{j}, t_{j}, M r_{j}\right)\right)}{\omega\left(\Delta\left(X_{j}, t_{j}, M r_{j}\right)\right)}\left[(1+\epsilon) \frac{\omega\left(\Delta\left(X_{j}, t_{j}, M r_{j}\right)\right)}{\sigma\left(\Delta\left(X_{j}, t_{j}, M r_{j}\right)\right)}\right. \\
& \left.+\epsilon^{3 / 4} M^{\frac{3}{4}(n+1)} \frac{\omega\left(\Delta\left(X_{j}, t_{j}, r_{j}\right)\right)}{\sigma\left(\Delta\left(X_{j}, t_{j}, r_{j}\right)\right)}\right] \\
& \leq\left(1+C \epsilon^{3 / 4} M^{\frac{3}{4}(n+1)}\right) .
\end{aligned}
$$

Furthermore,

$$
I_{j} \leq(1-\epsilon)^{-1}\left(1-C M^{n+1} \epsilon\right)^{-1} \text {. }
$$

In total it follows there exist two functions $A(\epsilon)$ and $B(\epsilon)$ such that if $j \geq j_{0}$ then

$$
A(\epsilon) \leq I_{j} \leq B(\epsilon)
$$

Furthermore, $A(\epsilon) \rightarrow 1$ and $B(\epsilon) \rightarrow 1$ as $\epsilon \rightarrow 0$. Continuing we have

$$
(1-\epsilon) I_{j} \int_{\hat{G}_{j}} \phi d \sigma_{j} \leq \int_{\hat{G}_{j}} \phi k_{j} d \sigma_{j} \leq(1+\epsilon) I_{j} \int_{\hat{G}_{j}} \phi d \sigma_{j} .
$$


Note that

$$
\begin{aligned}
\int_{\partial \Omega_{j}} \phi d \omega_{j} & =\int_{\partial \Omega_{j}} \phi k_{j} d \sigma_{j}=\int_{\hat{G}_{j}} \phi k_{j} d \sigma_{j}+\int_{\hat{F}_{j}} \phi k_{j} d \sigma_{j} \\
& \leq(1+\epsilon) I_{j} \int_{\hat{G}_{j}} \phi d \sigma_{j}+\|\phi\|_{\infty} \omega_{j}\left(\hat{F}_{j}\right) \\
& \leq(1+\epsilon) I_{j} \int_{\partial \Omega_{j}} \phi d \sigma_{j}+C_{M} \epsilon^{3 / 4}\|\phi\|_{\infty} .
\end{aligned}
$$

Similarly

$$
\begin{aligned}
\int_{\partial \Omega_{j}} \phi d \omega_{j} & \geq \int_{\hat{G}_{j}} \phi k_{j} d \sigma_{j} \geq(1-\epsilon) I_{j} \int_{\hat{G}_{j}} \phi d \sigma_{j} \\
& =(1-\epsilon) I_{j} \int_{\partial \Omega_{j}} \phi d \sigma_{j}-(1-\epsilon) I_{j} \int_{\hat{F}_{j}} \phi d \sigma_{j} \\
& \geq(1-\epsilon) I_{j} \int_{\partial \Omega_{j}} \phi d \sigma_{j}-C_{M}(1-\epsilon) I_{j} \epsilon\|\phi\|_{\infty} .
\end{aligned}
$$

Based on this we can conclude that

$$
\begin{aligned}
& \int_{\partial \Omega_{j}} \phi d \omega_{j} \leq(1+\epsilon) B(\epsilon) \int_{\partial \Omega_{j}} \phi d \sigma_{j}+C_{M} \epsilon^{3 / 4}\|\phi\|_{\infty}, \\
& \int_{\partial \Omega_{j}} \phi d \omega_{j} \geq(1-\epsilon) A(\epsilon) \int_{\partial \Omega_{j}} \phi d \sigma_{j}-C_{M}(1-\epsilon) B(\epsilon) \epsilon\|\phi\|_{\infty} .
\end{aligned}
$$

Hence

$$
\int_{\partial \Omega_{\infty}} \phi d \omega_{\infty}=\lim _{j \rightarrow \infty} \int_{\partial \Omega_{j}} \phi d \omega_{j}=\lim _{j \rightarrow \infty} \int_{\partial \Omega_{j}} \phi d \sigma_{j}
$$

Lemma 18. Assume that $\log k^{i}\left(\hat{X}^{i}, \hat{t}^{i}, \cdot\right) \in \operatorname{VMO}(d \sigma)$ for $i=1$ and $i=2$ and let $\omega_{j}^{i} \rightarrow \omega_{\infty}^{i}, u_{j}^{i} \rightarrow u_{\infty}^{i}$ in the sense of Lemma 16. There exists a constant $C$ such that for all $(Y, s) \in \Omega_{\infty}^{i}$

$$
u_{\infty}^{i}(Y, s) \leq C \delta_{\infty}^{i}(Y, s) .
$$

Proof. Let $(X, t) \in \partial \Omega_{\infty}^{i}$ and $r$ be such that $A_{r}(X, t)=(Y, s)$. By Lemma 13 and $16, u_{\infty}^{i}$ can be considered as the Green function with pole at + infinity and $\omega_{\infty}^{i}$ as the associated caloric measure. By Lemma 7 and Lemma 8, combined with Lemma 17, we have

$$
r^{n} u_{\infty}^{i}\left(A_{r}(X, t)\right) \sim \omega_{\infty}^{i}\left(\Delta(X, t, r) \leq C r^{n+1} .\right.
$$


As $r \sim \delta_{\infty}^{i}(Y, s)$ we can therefore conclude that there exists a universial constant $C$ such that $u_{\infty}^{i}(Y, s) \leq C \delta_{\infty}^{i}(Y, s)$ for all $(Y, s) \in \Omega_{\infty}^{i}$.

\subsection{Classification of global solutions and the proof of Theorem 1}

Lemma 19. Assume that the assumptions in Lemma 17 are fulfilled and, using the notation of Lemma 16, $u_{j}^{i} \rightarrow u_{\infty}^{i}$ for $i \in\{1,2\}$. Define for $(Y, s) \in \mathrm{R}^{n+1}$, $u_{\infty}(Y, s)=u_{\infty}^{1}(Y, s)-u_{\infty}^{2}(Y, s)$, where $u_{\infty}^{1}(Y, s) \equiv 0$ in $\Omega_{\infty}^{2}, u_{\infty}^{2}(Y, s) \equiv 0$ in $\Omega_{\infty}^{1}$. Then $u_{\infty}$ is a linear function in the space variables and $\Omega_{\infty}$ is a half space containing a line parallel to the time-axis.

Proof. Applying Lemma 17 we can conclude that $\omega_{\infty}^{1} \equiv \omega_{\infty}^{2}$ and that for all $\phi \in C_{0}^{\infty}\left(\mathrm{R}^{n+1}\right)$

$$
\int_{\partial \Omega_{\infty}^{i}} \phi(Y, s) d \omega_{\infty}^{i}(Y, s)=\int_{\Omega_{\infty}^{i}} u_{\infty}^{i}(Y, s)\left(\Delta-\partial_{s}\right) \phi(Y, s) d Y d s .
$$

Hence

$$
\begin{aligned}
\int_{\mathrm{R}^{n+1}} & u_{\infty}(Y, s)\left(\Delta-\partial_{s}\right) \phi(Y, s) d Y d s \\
= & \int_{\Omega_{\infty}^{1}} u_{\infty}^{1}(Y, s)\left(\Delta-\partial_{s}\right) \phi(Y, s) d Y d s \\
& \quad-\int_{\Omega_{\infty}^{2}} u_{\infty}^{2}(Y, s)\left(\Delta-\partial_{s}\right) \phi(Y, s) d Y d s \\
= & 0 .
\end{aligned}
$$

As $u_{\infty}$ is continuous, it is weakly adjoint caloric in $\mathrm{R}^{n+1}$ and therefore adjoint caloric in $\mathrm{R}^{n+1}$. By a change of the time direction we can assume that $u_{\infty}$ is caloric in $\mathrm{R}^{n+1}$. We also note that $u_{\infty}(0,0)=0$. By standard estimates for the heat equation we have that

$$
\max _{C_{r / 2}(0,0)}\left|D_{Z}^{k} D_{\tau}^{l} u_{\infty}(Z, \tau)\right| \leq \frac{C_{k l}}{r^{k+2 l}} \max _{C_{r}(0,0)}\left|u_{\infty}(Z, \tau)\right| .
$$

According to Lemma $15, \Omega_{\infty}$ defines a two-sided parabolic NTA-domain, and hence $A_{r}^{1}(X, t)$ and $A_{r}^{2}(X, t)$ are well-defined for $(X, t) \in \partial \Omega$ and $r>$ 0 . Using this points of reference we have by the backward in time Harnack principle in Lemma 8 that

$$
\max _{C_{r}(0,0)}\left|u_{\infty}(Z, \tau)\right| \leq C \max \left\{u_{\infty}^{1}\left(A_{r}^{1}(0,0)\right), u_{\infty}^{2}\left(A_{r}^{2}(0,0)\right)\right\} .
$$

Using Lemma 7 and Lemma 8 we have

$$
\frac{r^{n} u_{\infty}^{1}\left(A_{r}^{1}(0,0)\right)}{\omega_{\infty}^{1}(\Delta(0,0, r))} \sim \frac{r^{n} u_{\infty}^{2}\left(A_{r}^{2}(0,0)\right)}{\omega_{\infty}^{2}(\Delta(0,0, r))} \sim 1 .
$$


Hence as $\omega_{\infty}^{1} \equiv \omega_{\infty}^{2}$ we can conclude that $u_{\infty}^{1}\left(A_{r}^{1}(0,0)\right) \sim u_{\infty}^{2}\left(A_{r}^{2}(0,0)\right)$. According to Lemma $18, u_{\infty}^{1}\left(A_{r}^{1}(0,0)\right) \leq C r$. In total we can therefore conclude that

$$
\max _{C_{r / 2}(0,0)}\left|D_{Z}^{k} D_{\tau}^{l} u_{\infty}(Z, \tau)\right| \leq \frac{C_{k l}}{r^{k+2 l}} u_{\infty}^{1}\left(A_{r}^{1}(0,0)\right) \leq \frac{C_{k l}}{r^{k+2 l-1}} .
$$

By letting $r \rightarrow \infty$ we get $D_{Z}^{k} D_{\tau}^{l} u_{\infty}(Z, \tau)=0$ for all $(Z, \tau) \in \mathrm{R}^{n+1}$ and all $(k, l)$ such that $k+2 l-1>0$. We can therefore conclude that $u_{\infty}$ is in fact a linear function in the space variables and $\Omega_{\infty}$ is a half space containing a line parallel to the time-axis.

We can now prove Theorem 1 using Lemma 19. As $\partial \Omega$ separates $\mathrm{R}^{n+1}$ there exists, according to Definition $3, \delta_{0}>0$ such that given any $(\tilde{X}, \tilde{t}) \in \partial \Omega$, $R>0$, there exists a $n$ dimensional plane $\hat{P}=\hat{P}(\tilde{X}, \tilde{t}, R)$, containing $(\tilde{X}, \tilde{t})$ and a line parallel to the $t$ axis, having unit normal $\hat{n}=\hat{n}(\tilde{X}, \tilde{t}, R)$ such that

$$
\begin{aligned}
& \left\{(Y, s)+r \hat{n} \in C_{R}(\tilde{X}, \tilde{t}):(Y, s) \in \hat{P}, r>\delta_{0} R\right\} \subset \Omega, \\
& \left\{(Y, s)-r \hat{n} \in C_{R}(\tilde{X}, \tilde{t}):(Y, s) \in \hat{P}, r>\delta_{0} R\right\} \subset \mathrm{R}^{n+1} \backslash \Omega .
\end{aligned}
$$

We therefore introduce the quantity

$$
\Theta(\tilde{X}, \tilde{t}, R):=\frac{1}{R} \inf _{\hat{P}} D\left[\partial \Omega \cap C_{R}(\tilde{X}, \tilde{t}), \hat{P} \cap C_{R}(\tilde{X}, \tilde{t})\right]
$$

where the infimum is taken over all $n$ dimensional planes $\hat{P}=\hat{P}(\tilde{X}, \tilde{t}, R)$, containing $(\tilde{X}, \tilde{t})$ and a line parallel to the $t$ axis. For any compact set $K \subset \mathbf{R}^{n+1}$ we also introduce

$$
\Theta_{K}(R):=\sup _{(\tilde{X}, \tilde{t}) \in K} \Theta(\tilde{X}, \tilde{t}, R) .
$$

If $(X, t) \in \partial \Omega, r>0$, then the statement that $C_{r}(X, t) \cap \partial \Omega$ is Reifenberg flat with vanishing constant in the parabolic sense is equivalent to the statement that

$$
\lim _{\hat{r} \rightarrow 0} \Theta_{C_{r}(X, t) \cap \partial \Omega}(\hat{r})=0 .
$$

To prove Theorem 1 we assume, using the notation of the theorem, that $(X, t) \in$ $\partial \Omega, \hat{t}^{2}>t+4 r^{2}$ and that

$$
\lim _{\hat{r} \rightarrow 0} \Theta_{C_{r}(X, t) \cap \partial \Omega}(\hat{r})=\beta
$$

for some $\beta>0$. We intend to prove that this is impossible and that $\beta=0$. Let $\left(X_{j}, t_{j}\right) \in C_{r}(X, t) \cap \partial \Omega,\left(X_{j}, t_{j}\right) \rightarrow(\hat{X}, \hat{t}) \in C_{r}(X, t) \cap \partial \Omega$ and let $r_{j}$ be a sequence of real numbers tending to zero such that

$$
\lim _{j \rightarrow \infty} \Theta\left(X_{j}, t_{j}, r_{j}\right)=\beta .
$$


By a translation argument we can without loss of generality assume that $(\hat{X}, \hat{t})=(0,0)$ and that $(0,0) \in C_{r}(X, t) \cap \partial \Omega$. Define the domains,

$$
\Omega_{j}^{i}=\left\{\left(r_{j}^{-1}\left(X-X_{j}\right), r_{j}^{-2}\left(t-t_{j}\right)\right):(X, t) \in \Omega^{i}\right\} .
$$

Then according to Lemma 15 we can assume that $\Omega_{j}^{i} \rightarrow \Omega_{\infty}^{i}, \partial \Omega_{j}^{i} \rightarrow \partial \Omega_{\infty}^{i}$ in the parabolic Hausdorff distance sense uniformly on compact subsets of $\mathrm{R}^{n+1}$. Furthermore, $\Omega_{\infty}=\Omega_{\infty}^{1}$ and $\Omega_{\infty}^{2}=\mathrm{R}^{n+1} \backslash \bar{\Omega}_{\infty}$ are parabolic NTA-domains and $\partial \Omega_{\infty}$ separates $R^{n+1}$. We can furthermore apply Lemma 16, Lemma 17 and Lemma 19 in order to conclude that $\Omega_{\infty}$ is a half space containing a line parallel to the time-axis. Still our assumption above give at hand that

$$
\Theta_{\infty}(0,0,1):=\beta>0
$$

where $\Theta_{\infty}(0,0,1)$ is defined w.r.t. $\partial \Omega_{\infty}$. Clearly this is a contradiction and we can conclude that $C_{r}(X, t) \cap \partial \Omega$ is Reifenberg flat with vanishing constant.

\section{REFERENCES}

1. Alt, H. W., Caffarelli, L., Existence and regularity for a minimum problem with free boundary, J. Reine Angew. Math. 325 (1981), 105-144.

2. Athanasopoulos, I., Caffarelli, L., Salsa, S., Regularity of the free boundary in parabolic phase transition problems, Acta Math. 176 (1996), no. 2, 245-282.

3. Athanasopoulos, I., Caffarelli, L., Salsa, S., Caloric functions in Lipschitz domains and the regularity of solutions to phase transition problems, Ann. of Math. (1) 143 (1996), no. 3, 463-484.

4. Doob, J. L., Classical Potential Theory and its Probablilistic Counterpart, Grundlehren Math. Wiss. 262 (1984).

5. Fabes E., and Safonov, M., Behaviour near the boundary of positive solutions of second order parabolic equations, J. Fourier Anal. Appl. 3 (1997), 871-882.

6. Fabes, E., Safonov, M., Yuan, Y., Behaviour near the boundary of positive solutions of second order parabolic equations II, Trans. Amer. Math. Soc. 351 (1999), 4947-4961.

7. Garnett, J. B., Jones, P. W., The distance in BMO to $L^{\infty}$, Ann. of Math. 108 (1978), 373-393.

8. Garcia-Cuerva, J., Rubio de Francia, J. L., Weighted Norm Inequalities and Related Topics, North-Holland Math. Stud. 116 (1985).

9. Hofmann S., and Lewis, J., Solvability and representation by caloric layer potentials in timevarying domains, Ann. of Math. 144 (1996), 349-420.

10. Hofmann, S., Lewis, J., and Nyström, K., Existence of big pieces of graphs for parabolic problems, Ann. Acad. Sci. Fenn. Math. 28 (2) (2003), 355-384.

11. Hofmann, S., Lewis, J., and Nyström, K., Caloric measure in parabolic flat domains, Duke Math. J. 122 (2) (2004), 281-345.

12. Kenig, C., and Toro, T., Harmonic measure on locally flat domains, Duke Math. J. 87 (1997), 501-551.

13. Kenig, C., and Toro, T., Free boundary regularity for harmonic measure and Poisson kernels, Ann. of Math. 150 (1999), 369-454.

14. Kenig, C., and Toro, T., Poisson kernel characterization of Reifenberg flat chord arc domains, Ann. Sci. École Norm. Sup. (to appear). 
15. Kenig, C., and Toro, T., Free boundary regularity below the continuous threshold: 2-phase problems, preprint.

16. Kaufmann, R., and Wu, J. M., Parabolic measure on domains of class $\mathrm{Lip}_{1 / 2}$, Compositio Math. 65 (1988), 201-207.

17. Lewis, J., and Murray, M., The method of layer potentials for the heat equation in time-varying domains, Mem. Amer. Math. Soc. 114 (1995), no. 545.

18. Lewis, J., and Silver, J., Parabolic measure and the Dirichlet problem for the heat equation in two dimensions, Indiana Univ. Math. J. 37 (1988), 801-839.

19. Nyström, K., The Dirichlet problem for second order parabolic operators, Indiana Univ. Math. J. 46 (1997), 183-245.

20. Nyström, K., Caloric measure and Reifenberg flatness, to appear.

DEPARTMENT OF MATHEMATICS

UMEÅ UNIVERSITY

S-90187 UMEA

SWEDEN

E-mail: kaj.nystrom@math.umu.se 